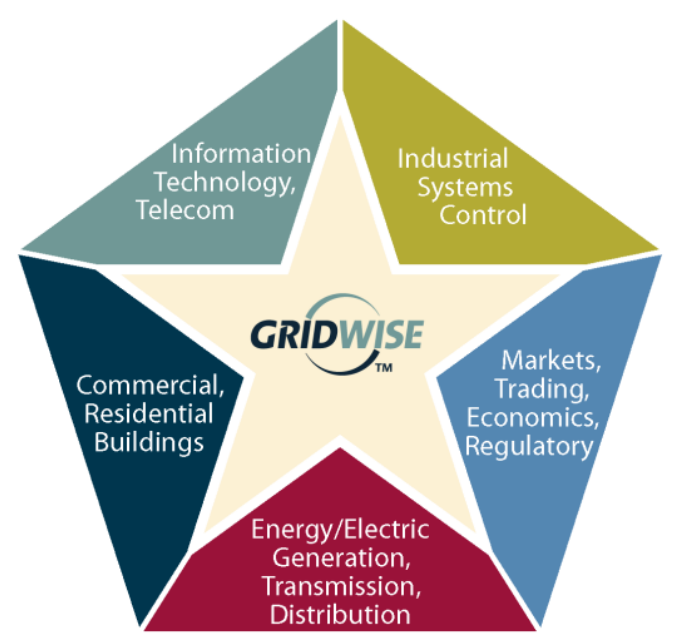

\title{
Smart Grid Interoperability Maturity Model Beta Version
}

Prepared by

The GridWise Architecture Council

December 2011

\section{About this Document}

The GridWise Architecture Council was formed by the U.S. Department of Energy to promote and enable interoperability among the many entities that interact with the electric power system. This balanced team of industry representatives proposes principles for the development of interoperability concepts and standards. The Council provides industry guidance and tools that make it an available resource for smart grid implementations. In the spirit of advancing interoperability of an ecosystem of smart grid devices and systems, this document presents a model for evaluating the maturity of the artifacts and processes that specify the agreement of parties to collaborate across an information exchange interface. You are expected to have a solid understanding of large, complex system integration concepts and experience in dealing with software component interoperation. Those without this technical background should read the Executive Summary for a description of the purpose and contents of the document. Other documents, such as checklists, guides, and whitepapers, exist for targeted purposes and audiences. Please see the www.gridwiseac.org website for more products of the Council that may be of interest to you.

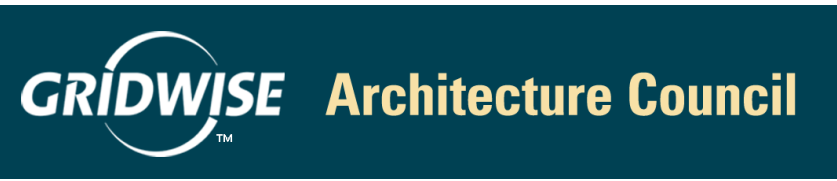




\section{RIGHT TO DISTRIBUTE AND CREDIT NOTICE}

This material was created by the GridWise ${ }^{\circledR}$ Architecture Council and is available for public use and distribution. Please include credit in the following manner: The Smart Grid Interoperability Maturity Model is a work of the GridWise Architecture Council.

\section{DISCLAIMER}

This document represents a step toward establishing a model for assessing and promoting interoperability maturity. It forms a basis for engaging system integration experts in discussions that lead to improvements in this early material. It was prepared by the GridWise Architecture Council, interested collaborators of the Council, and employees of Battelle Memorial Institute (Battelle) as an account of sponsored research activities. Neither Client nor Battelle nor any person acting on behalf of either:

MAKES ANY WARRANTY OR REPRESENTATION, EXPRESS OR IMPLIED, with respect to the accuracy, completeness, or usefulness of the information contained in this report, or that the use of any information, apparatus, process, or composition disclosed in this report may not infringe privately owned rights; or

Assumes any liabilities with respect to the use of, or for damages resulting from the use of, any information, apparatus, process, or composition disclosed in this report.

Reference herein to any specific commercial product, process, or service by trade name, trademark, manufacturer, or otherwise, does not necessarily constitute or imply its endorsement, recommendation, or favoring by the GridWise Architecture Council or Battelle. The views and opinions of authors expressed herein do not necessarily state or reflect those of Battelle. 


\section{Executive Summary}

The integration of automation associated with electricity resources (including transmission and distribution automation and demand-side resources operated by end-users) is important to supporting greater efficiencies and incorporating variable renewable resources and electric vehicles into the power system. The integration problems faced by this community are analogous to those faced in the health industry, emergency services, and other complex communities with many stakeholders. To highlight this issue and encourage communication and the development of a smart grid interoperability community, the GridWise ${ }^{\circledR}$ Architecture Council (GWAC) created an Interoperability Context-Setting Framework (see Figure S.1). This "conceptual model" has been helpful to explain the importance of organizational alignment in addition to technical and informational interface specifications for "smart grid" devices and systems. As a next step to building a community sensitive to interoperability, the GWAC has been developing a smart grid interoperability maturity model (SG IMM) borrowing from work done by others to address similar circumstances. The model provides a means to measure status and progress, analyze gaps, and prioritize efforts to improve the situation. The objective is to create a tool, or set of tools, that encourages a culture of interoperability in the smart grid community.

\section{So What?}

Interoperability enables intelligent devices and systems to connect and work securely and effectively with little or no inconvenience to the user. When the scale of information and communications technology (ICT) connectivity is as big as the electric power system, the multipliers are large and the benefits of improving interoperability are manifold. The benefits include,

$\checkmark$ reduced integration cost $\checkmark$ reduced cost to operate $\checkmark$ reduced capital ICT cost $\checkmark$ reduced installation cost $\checkmark$ reduced upgrade cost $\checkmark$ better security management $\checkmark$ more choice in products $\checkmark$ more price points and features

Realizing these benefits is critical for establishing the business case and the value proposition for decision-makers who review smart grid deployment proposals and enable smart grid concepts to flourish.

Figure S.1: GWAC Interoperability Context-Setting Framework

\section{An Interoperability Improvement Process}

To achieve interoperable products and systems, the interfaces between them need to be clearly specified with well-defined responsibilities and expectations understood between the interacting parties. The SG IMM was inspired by work done by the National E-Health Transition Authority of Australia (NEHTA), which articulated the adaptation of capability maturity model concepts from the Software Engineering Institute at Carnegie Mellon University (SEI) to go beyond a corporate enterprise and address the need to exchange medical information between the multitudes of stakeholders in the health community. The issue in the health system, as well as in the electric system, is developing and improving interface

\section{Page 3 of 46}


standards that allow the many players to easily connect, effectively communicate, and enable automation to deliver value in new ways.

An ecosystem of products and systems that collaborate to provide useful services begins with pairwise communication between parties that may be ad hoc at first, but eventually are documented. Project-level interface specifications may be shared with other parties as the concepts and value propositions mature. Communities of interested organizations may come together under the umbrella of a standards organization to develop one or more standards, and implementations that support these standards may eventually be tested and certified by testing authorities to meet the requirements of the standards. As technology and value propositions advance, the performance of the standard may be measured; the results can be used to identify and prioritize changes to the standards, resulting in new versions of the standards and associated tests. These steps represent progress in the maturity of the interoperability of the products and systems that service the community. By developing processes that move the community to higher levels of maturity, the discipline to improve interoperability can advance.

\section{A Structure for Exploring the Full Dimensions of Interoperability}

A model for this progression in software development, called a capability maturity model, has been articulated by the SEI and is widely embraced as a process improvement approach for developing highquality software and systems. The five maturity levels in this model are used as a maturity measure for interoperability by the SG IMM. Because the GWAC Interoperability Context-Setting Framework [5] provides a mechanism for exploring the multiple dimensions of interoperability, the SG-IMM used this model as an organized landscape for identifying interoperability goals. The SG IMM transforms these goals into a series of metrics. Statements about the characteristics expected at each maturity level are then used as tests for evaluating the maturity of an interface specification of interest. Figure S.2 depicts a simplified view of the framework with the maturity levels incorporated as a third dimension.

The SG IMM aims to enhance the quality of interface specifications used in the smart grid community. The efforts required to define good metrics that can indicate the level of interoperability maturity are justified by the efficiency of the integration between the different devices and systems and the reliability of the integration experience.

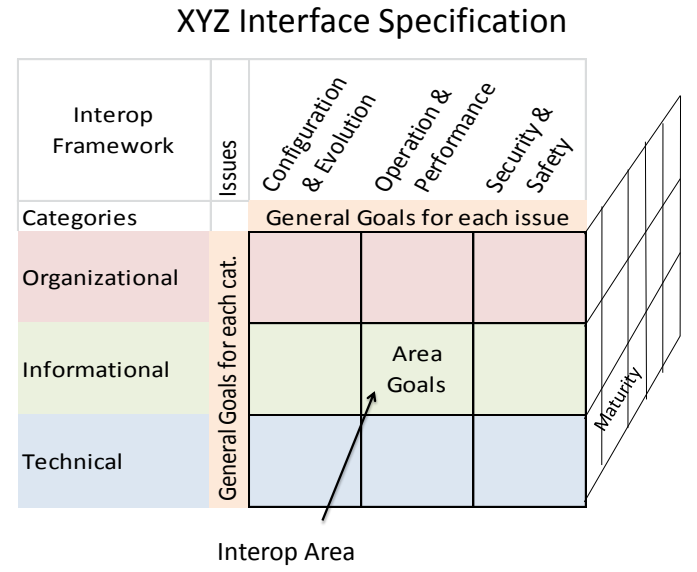

Figure S.2: Simplified Interoperability Framework for SG IMM 


\section{Moving Forward}

The GWAC is releasing an initial (beta) draft of this model to introduce the concepts and solicit participation by interested stakeholders to develop these ideas into a workable model with an assessment methodology and tools. As such, the statements deserve careful scrutiny. Those who have contributed to this work recognize the need for improvement. Besides advancing the SG IMM to a usable product, more participation is needed to socialize the concepts and help with the adoption of the ultimate model by our smart grid community.

With this in mind, this draft model is undergoing early tests in a set of trials. Two such trials are now underway and the team is soliciting the involvement of other application areas to test the initial evaluation tool. In addition, a workshop is being planned to bring together smart grid integration and process improvement experts for a review of the SG IMM beta in the first part of 2012. The results of the workshop and the trials are expected to provide necessary feedback so that the beta model can be advanced to a Version 1.0 of the SG IMM. Additional experience and new participants are also expected to reveal new uses for the model and different directions that evaluation methods and tools can take. Other documents and efforts will hopefully follow as these ideas cultivate interoperability in the smart grid deployment culture.

The SG IMM is also not the only work product advancing maturity in the smart grid community. Cyber security approaches are also maturing, and a functional maturity model for electric utilities has already been established called the Smart Grid Maturity Model (SGMM), which also happens to be managed by the SEI. Discussions have been initiated with the U.S. Department of Energy and the SEI on the potential to coordinate the SEI SGMM efforts with the GWAC SG IMM. Based on the complementary nature of the models and their intent to advance maturity in the smart grid community, collaboration should be beneficial.

In summary, the mission of the GridWise Architecture Council (GWAC) is to enable interoperability among the many entities that interact with the electric power system. The development of the Smart Grid Interoperability Maturity Model (SG IMM) represents a crucial step toward defining metrics for determining the current quality and levels of interoperability among the interacting entities. It defines a model for measuring the interoperability aspects of the interfaces between various entities in the electric power system. 


\section{About GridWise ${ }^{\circledR}$ and the Architecture Council}

The GridWise vision rests on the premise that information technology will revolutionize planning and operation of the electric power grid just as it has transformed business, education and entertainment. Information technology will form the "nervous system" that integrates new distributed technologiesdemand response, distributed generation and storage - with traditional grid generation, transmission and distribution assets. Responsibility for managing the grid will be shared by a "society" of devices and system entities.

The GridWise Architecture Council's mission is to enable all elements of the electric system to interact. We are an independent body that believes tomorrow's electric infrastructure can be more efficient and secure by integrating information technology and e-commerce with distributed intelligent networks and devices. To achieve this vision of a transformed electric system, the GridWise Architecture Council is defining the principles for interaction among the information systems that will effectively and dynamically operate the grid. The Council, which is supported by the U.S. Department of Energy, includes thirteen representatives from electric energy generation and delivery, industrial systems control, building automation, information technology and telecommunications, and economic and regulatory policy.

The GridWise Architecture Council is shaping the guiding principles of a highly intelligent and interactive electric system - one ripe with decision-making information exchange and market-based opportunities. This high-level perspective provides guidelines for interaction between participants and interoperability between technologies and automation systems. We seek to:

- develop and promote the policies and practices that will allow electric devices, enterprise systems, and their owners to interact and adapt as full participants in system operations.

- shape the principles of connectivity for intelligent interactions and interoperability across all automation components of the electric system from end-use systems, such as buildings or heating, ventilation, and air conditioning (HVAC) systems, to distribution, transmission and bulk power generation.

- address issues of open information exchange, universal grid access, decentralized grid communications and control, and the use of modular and extensible technologies that are compatible with the existing infrastructure.

The Council is neither a design team nor a standards-making body. Our role is to bring the right parties together to identify actions, agreements, and standards that enable significant levels of interoperation between automation components. We act as catalysts to outline a philosophy of inter-system operation that preserves the freedom to innovate, design, implement and maintain each organization's role and responsibility in the electrical system.

Page 6 of 46

The Smart Grid Interoperability Maturity Model is a work of the GridWise ${ }^{\circledR}$ Architecture Council 


\section{Contents}

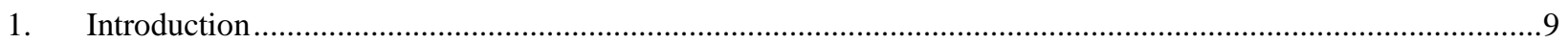

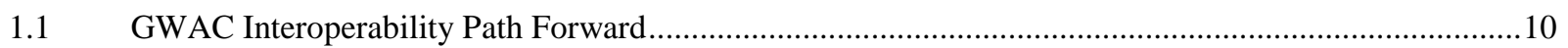

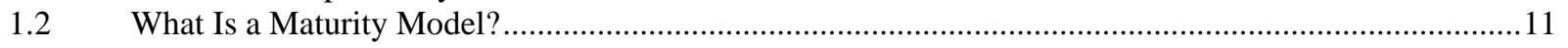

$1.3 \quad$ Why Develop an Interoperability Maturity Model? .........................................................................13

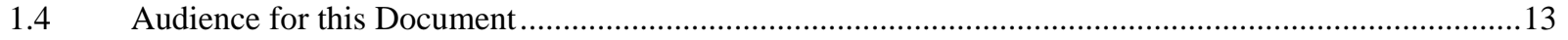

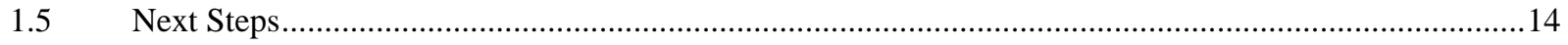

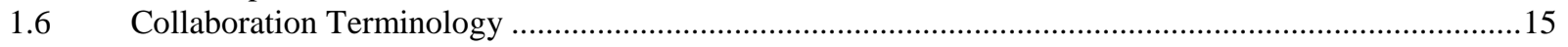

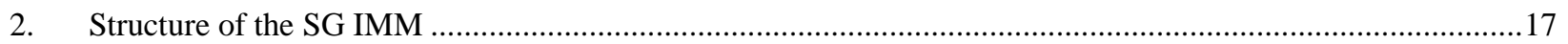

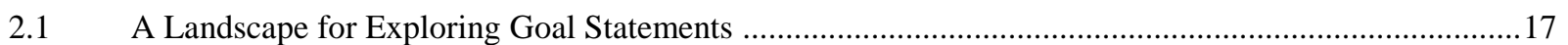

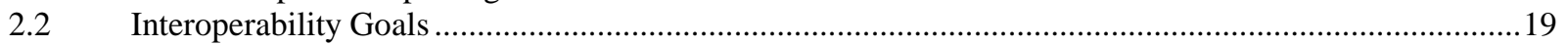

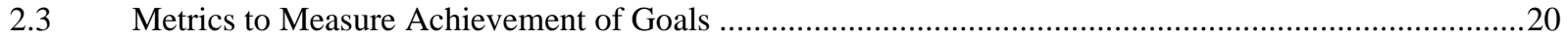

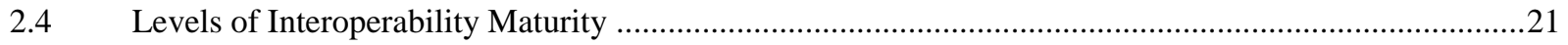

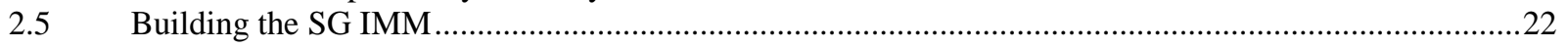

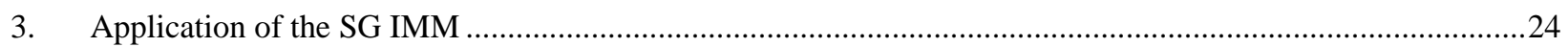

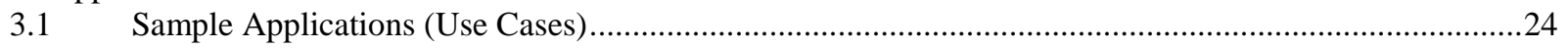

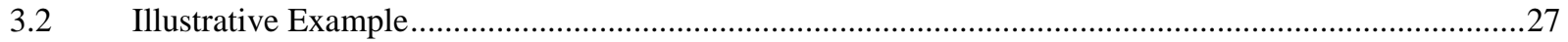

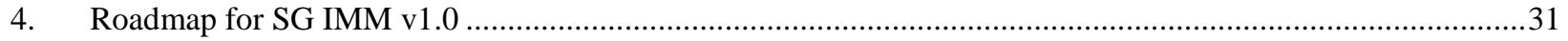

$4.1 \quad$ Trials

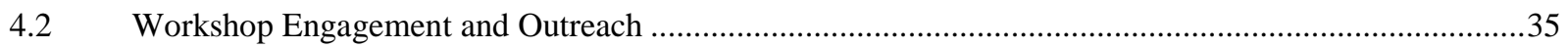

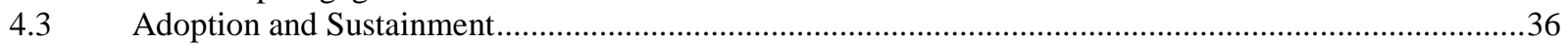

4.4 Relationship with Interoperability Testing \& Certification .................................................................. 36

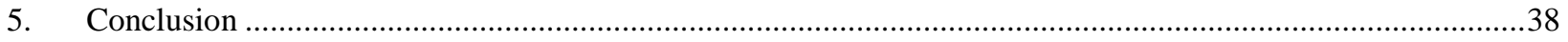

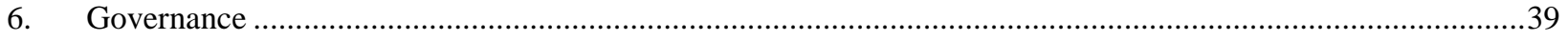

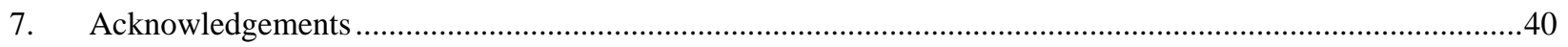

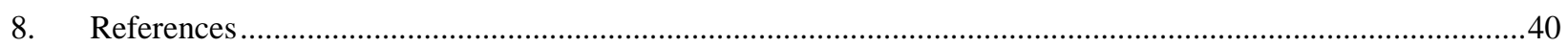

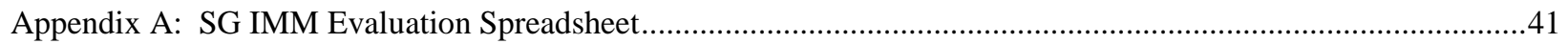

The Smart Grid Interoperability Maturity Model is a work of the GridWise ${ }^{\circledR}$ Architecture Council 


\section{Figures}

Figure S.1: GWAC Interoperability Context-Setting Framework …................................................... 3

Figure S.2: Simplified Interoperability Framework for SG IMM..................................................... 4

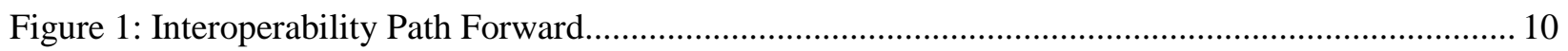

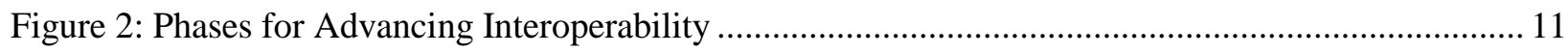

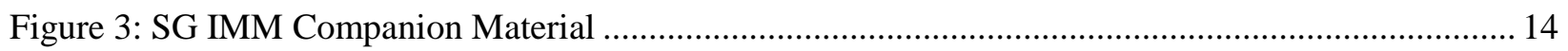

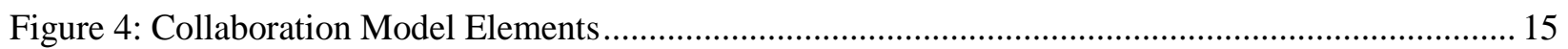

Figure 5: Interoperability Context-Setting Framework..................................................................... 17

Figure 6: Simplified Interoperability Framework for SG IMM ............................................................ 18

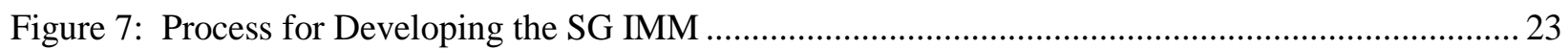

Figure 8: PNWSGDP Interacting Nodes and Logical Communication Structure .................................... 33

Figure 9: Transactive Signal Interfaces between Participants ................................................................. 33

\section{Tables}

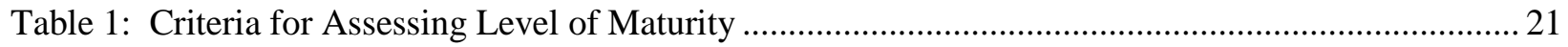

Table 2: SG IMM Test and Certification Maturity Statements............................................................. 37 


\section{Introduction}

Improving interoperability is critical to the achievement of the objectives associated with enabling smart grid capabilities. Lessons from supply chains in other sectors indicate that advancements in their ability to address interoperability issues produce significant benefits [1]. To do this requires that the stakeholders in this community engage in actions to continually improve interoperability; an interoperability maturity model for the smart grid community is proposed for this purpose [2].

Interoperability within and between electricity system stakeholder organizations can benefit from the application of a model for defining and evaluating the process to achieve interoperability. An important aspect of this model is its ability to focus on the automation interfaces that connect organizations and address the incremental nature of improving interoperability as implementations evolve over time.

Deployments of smart grid capabilities need to address the integration challenges that go beyond single organizations or enterprises to address the interactions that span organizational entities. The GridWise Architecture Council (GWAC) was formed to promote and enable interoperability among the many entities that interact to make up the electric power system. While guidance, methods, and tools to enable interoperability can benefit all types of integration, the emphasis of the GWAC work is to enable interoperability between organizations. To this end, the GWAC has championed a constitution of interorganizational interoperability principles [3]. Consistent with these principles, the GWAC developed a path forward for engaging the smart grid community of stakeholders to appreciate interoperability and share concepts, organize interoperability needs and issues, debate and prioritize activities, and measure progress [4]. As a part of this approach they engaged the stakeholder community to establish an interoperability context-setting framework [5] to create a model for sharing interoperability concepts and issues.

Smart grid interoperability community development took a leap forward when the United States government enacted the Energy Independence and Security Act of 2007, which gave the National Institute of Standards and Technology (NIST) the directive to advance interoperability, standards, and model protocols to enable smart grid capabilities. In November, 2009, NIST formed the Smart Grid Interoperability Panel (SGIP) and encouraged smart grid stakeholders from all organizations associated with electric power to establish this community to advance interoperability through goals, gap analysis, and prioritized efforts to better address the challenges to integration.

As work on standards and best practices progresses, this work only address part of the need. Interoperability must deal with agreements between interacting parties who require the flexibility to address special arrangements and manage change over time. This requires a change in the culture of system integration. To help assess and encourage behavior that improves interoperability over time, process improvement methods and tools are proposed for development by and for the smart grid community.

The idea is to develop a smart grid interoperability maturity model (SG IMM) for application to communities of organizations engaged in smart grid product and project implementations. The model facilitates developing methods and processes that improve the integration and maintenance of the automation devices and systems. In addition, the model can be used to create tools for self-evaluation, resulting in recommendations for improving interoperability.

The U.S. Department of Energy also supports the Software Engineering Institute (SEI) at Carnegie Mellon University with a different type of maturity model to facilitate electric utility companies in advancing the functional aspects of smart grid deployment in their organizations [6]. The Smart Grid

Page 9 of 46 
Maturity Model (SGMM) provides a self-evaluation tool for utilities to assess various aspects of their enterprise in the adoption of information and communications technology to advance smart grid capabilities. The SG IMM and SGMM are complementary, in that the SG IMM intent is to advance interoperability between devices and systems that support the smart grid capabilities that the SGMM investigates.

This document presents the purpose, requirements, and an initial version of the SG IMM, including its applicability to smart grid stakeholders. It describes tools for applying the model to analyze the maturity of interfaces that support a community of interacting organizations as well as identifying practices that can help the community achieve its smart grid interoperability goals. While a SG IMM emphasizes interorganizational interoperability improvement, the concepts, methods, and tools can also be valuable within an individual organization. It should also be helpful in preparing an organization to more readily connect and interact with the smart grid community of interest (e.g., those stakeholders integrating electric vehicles for charging or those wishing to exchange system measurements for wide-area situational awareness).

\subsection{GWAC Interoperability Path Forward}

Advancing interoperability for smart grid applications is a community effort. No one has the keys to drive or dictate solutions across the thousands of organizations and interest groups involved with the electricity system. For this reason, the GWAC strives to engage all stakeholders and develop bridges across disciplines on a path to simplify integration. The GWAC Interoperability Path Forward whitepaper [4] outlined a path forward to improve interoperability. This is illustrated in Figure 1 and summarized in the following steps:

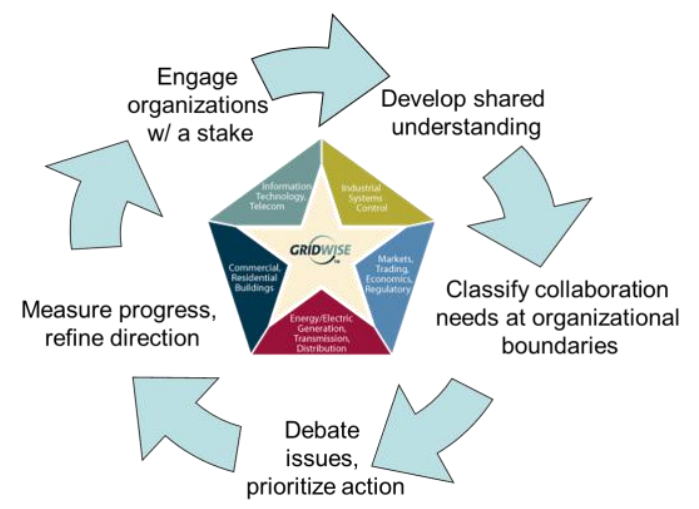

Figure 1: Interoperability Path Forward

1. Identify and engage the organizations, associations and regulatory spheres with stakes in the future of the electric system.

2. Develop a common understanding of interoperability and the various levels of interoperability.

3. Classify the information exchange needs, the collaboration required at organizational boundaries, and the issues impeding the advancement of interoperation.

4. Establish liaisons and working groups with influential organizations to debate interoperability issues in an open manner that disseminates important points for all to monitor, comment upon, and prioritize action (standards, policy, etc.).

5. Measure progress along this path and refine directions accordingly.

Progress has been made on each of these steps, but arguably the most difficult step has involved the measurement of progress with results that can help set a direction for future efforts. The SG IMM presents methodology and tools to help address the issue of measuring interoperability at the interface

Page 10 of 46 
level and, in the process, identify directions for improvement. Inspired by the process improvement aspects of the SEI's Capability Maturity Model for Integration (CMMI) [7] and the notions espoused by the National E-Health Transition Authority (NEHTA) [8] of an interoperability maturity model for the Australian health community, this maturity model for interoperability helps a smart grid community of collaborating organizations understand the maturity of their present processes to ease integration and see that interactions between devices and systems perform reliably. The primary intention of the model is for use as a self-assessment, planning, and process improvement tool. Hopefully such a model can also become a tool to help understand the progress that the smart grid community is making to enhance their collective maturity level to advance interoperability.

The GWAC mission of promoting interoperability as an enabler for smart grid deployment is unfolding as a series of phases that advance interoperability concepts, methods, and tools in the smart grid community. Figure 2 diagrams the steps taken since the formation of the GWAC. The formation stage was marked by the establishment of statements of principle defined in the Interoperability Constitution ratified by stakeholders from all corners of the electricity stakeholder base. As the smart grid community took shape, the Interoperability Context-Setting Framework provided a foundation of concepts and terms to facilitate discussion and action steps to improve interoperability. The SGIP, established by NIST, provides a forum for the smart grid community to advance the making and testing of interoperability standards. The SGIP embraced the Context-Setting Framework and continues to broaden awareness of interoperability as an essential concern of the smart grid community. The Smart Grid Interoperability Maturity Model rides on the shoulders of each of these achievements, and through a process improvement approach, endeavors to engender a culture of interoperability to serve smart grid deployments going forward.

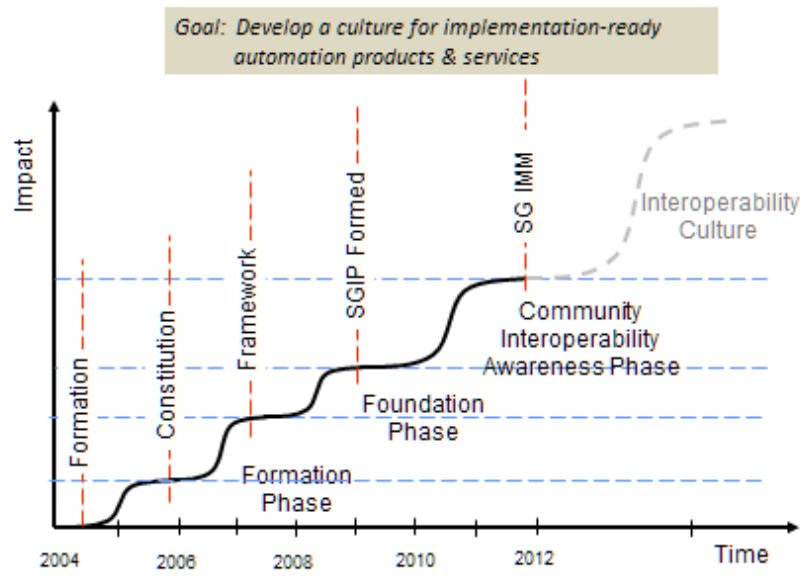

Figure 2: Phases for Advancing Interoperability

\subsection{What Is a Maturity Model?}

Quality assurance processes have been applied for decades. In the information technology space, the CMMI from the SEI [7] has a long history of explaining the concepts behind a capability maturity model and developing methodology and tools to help organizations evolve their processes in a continuous quality improvement lifecycle. 
Capability maturity models guide organizations in continuous process improvement to enhance the capability of a business to deliver on its objectives. The SEI has developed the methodology and tools to help guide an organization to reach new levels of capability in system integration. These tools can be used to identify the present state of processes within an organization, point out priority areas for improvement, and develop plans to address these areas.

While the concept of a maturity model is attractive to any smart grid project, the interfaces that interconnect organizations make many smart grid capabilities transformational from a community perspective and challenge the ability to directly apply the internal, project-orientated CMMI concepts.

NEHTA applied the concepts behind CMMI and adapted them to advancing the electronic exchange of information in the Australian health industry. The NEHTA Interoperability Maturity Model [8] emphasizes the need to improve the interorganizational exchange of health data: "E-health interoperability is defined as a continual ability of an organisation or a system to use or offer business/technical services from or to another organisation/system and accordingly, exchange information with other organisations/systems to achieve a specified purpose in a given context." An analogous notion can be transferred to the advancement of interoperability maturity in the smart grid community of stakeholders.

The CMMI identifies five maturity levels to support those groups using a staged representation. A staged representation provides a structured way to improve processes one stage at a time. The five maturity levels follow:

- Level 1: Initial—ad hoc \& chaotic

- Level 2: Managed - planned \& executed in accordance with policy

- Level 3: Defined - described in standards, procedures, tools, and methods

- Level 4: Quantitatively Managed-quantitative objectives for performance measurement and management

- Level 5: Optimizing - continually improving processes based on quantitative understanding of the causes of variation

NEHTA adapted the CMMI maturity notions to address the interorganizational issues that face their industry. The NEHTA model defines the following levels:

- Level 0: none

- $\quad$ No processes to support cross-organizational interoperability

- Level 1: Initial

- Community interoperability vision

- Policy makers delivering social benefits

- $\quad$ Ad-hoc use of standards

- Level 2: Managed

- Interoperability frameworks

- Community/national collaboration

- Governance in development

- Level 3: Defined

- "Community architecture"

- Interoperability governance defined

- Governed use of open standards

- Level 4: Quantitatively Managed

- Established certification program

- Community interface agreements monitoring

Page 12 of 46

The Smart Grid Interoperability Maturity Model is a work of the GridWise ${ }^{\circledR}$ Architecture Council 
- $\quad$ Level 5: Optimizing

- Continuous interoperability innovations

- Enables community/social goals

- Emergence, dynamics, adaptation

In the formation of the SG IMM, the body of work and concepts behind the CMMI and NEHTA work forms a compelling foundation for naming and defining maturity levels.

\subsection{Why Develop an Interoperability Maturity Model?}

Achieving interoperability requires a coordinated process among all the stakeholders. Advancing interoperability requires an improvement process. The purpose of this effort is to develop an SG IMM for application to communities of organizations engaged in smart grid product and project implementations. The model facilitates developing methods and processes that improve the integration and maintenance of the automation devices and systems at the community level. It intends to provide a means for assessing interoperability progress in the smart grid community and to encourage an interoperability-aware culture with individual and shared roadmaps for improvement. In addition, the model can be used to create tools for self-evaluation, resulting in the identification of gaps (descriptive) and recommendations for improving interoperability (prescriptive). The SG IMM emphasizes interorganizational interoperability improvement with focus on the automation interfaces between organizations. It should also be helpful to prepare an organization to more readily connect and interact with the smart grid community of interest. The community of interacting organizations that it supports distinguishes this effort from an enterprise integration maturity model. In the enterprise case, the possibility of enforcing an overall architecture and choice of technology exists. In a free marketplace, technology choice and design decisions must be respected between interacting organizations. The interfaces between them become business and technical contracts that enable flow of goods and services.

The main requirements of the SG IMM are that it be able to encourage the maturity of interorganizational integration. It must be simple and straightforward to be usable and useful. While interoperability is complex, the maturity model should be organized to be readily understood at a high level with details and complex issues being addressed as users move further into the model. The model should focus on the interface between interacting organizations' automation systems, understanding that while true interoperability extends into the implementations on either side of the interface, the interface agreement needs to reflect the expectations of all parties and repercussions for failure to perform.

The model and subsequent tools are not intended to be applied in a punitive fashion. The SG IMM is designed to encourage and enhance cooperation and evolution of maturity of all committed stakeholders rather than be used as a device to select one stakeholder over another or one set of organizations over another.

\subsection{Audience for this Document}

The SG IMM builds upon and leverages the structure and concepts already established in the Interoperability Context-Setting Framework and it borrows from the work of others, in particular, the CMMI and NEHTA work, to leverage well-developed ideas. In the creation of the beta version of the SG IMM, the authors presume an audience with a good understanding of interoperability, familiarity with the GWAC Interoperability Context-Setting Framework, and knowledge of product life-cycle process improvement concepts (such as those promulgated in the SEI's CMMI). People with this level of background should be reasonably able to understand the proposed ideas, be able to critically review them as proposals, and participate in reworking or refining the model so that it becomes a shared creation with tools that propagate and serve the diverse smart grid community. Subsequent work products are expected

Page 13 of 46

The Smart Grid Interoperability Maturity Model is a work of the GridWise ${ }^{\circledR}$ Architecture Council 
to engage the ultimate audience of this effort at a level that best communicates with each targeted segment.

The ultimate audience for a fully developed SG IMM addresses critical segments of the smart grid community including the following:

- smart grid integration domains, such as manufacturing, building, home, vehicle, and appliance integrators

- $\quad$ smart grid product and service suppliers

- owner/operators

- electric service providers

- regulators and policy makers

- assessors, analysts, and investigators

As a result, a set of companion material is envisaged to follow this document. The process to specify and develop future material requires the continued participation of the electricity community. Figure 3 provides a conceptual view of companion material envisaged to follow from the SG IMM base material. These works will include summaries for targeted audiences, technical papers that explain how the model may be applied and proposed approaches to address applications, trial applications, evaluation tools, surveys of the maturity of interfaces in various communities, and use case scenarios that provide examples of applying the SG IMM.

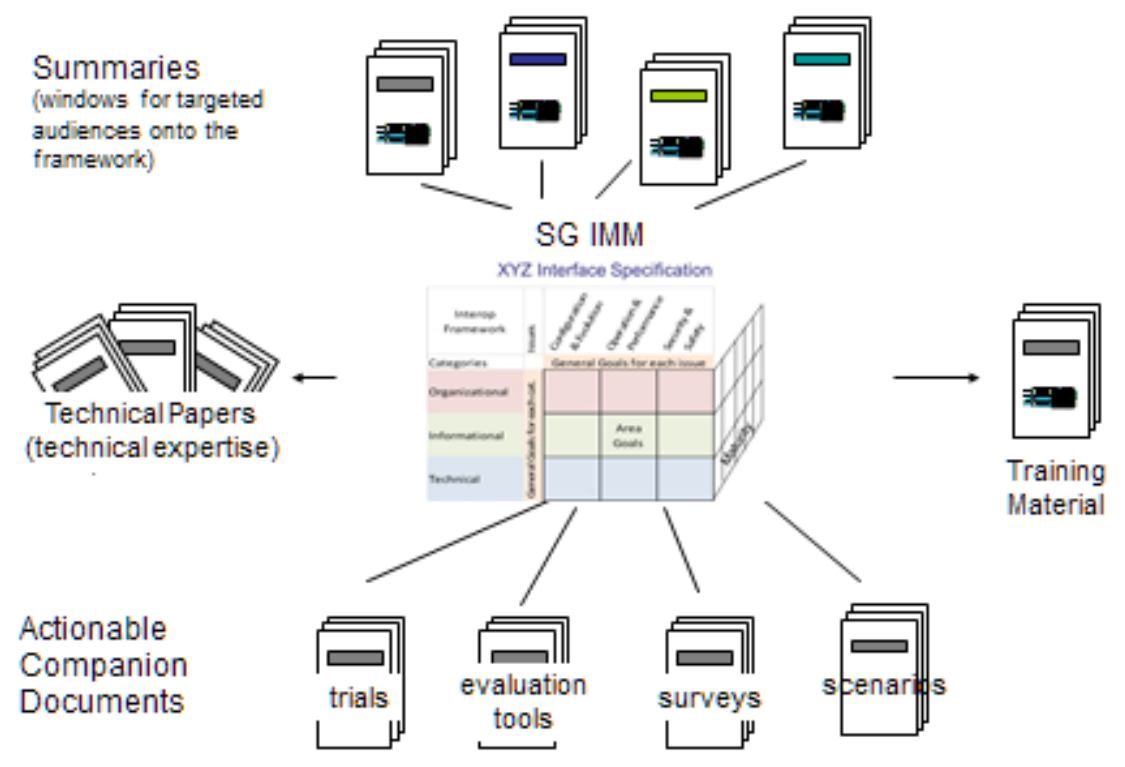

Figure 3: SG IMM Companion Material

\subsection{Next Steps}

With the development of the beta document, the GWAC intends to engage interoperability experts in the smart grid community in a dialogue about interoperability maturity assessment, the appropriateness of this initial model, and what shape it should take to best satisfy the needs of the community. Simultaneously, the initial model is expected be exercised by a few trial projects. Only by attempting to use the material will we understand whether it is realizing its objectives and how it can be refined to

Page 14 of 46

The Smart Grid Interoperability Maturity Model is a work of the GridWise ${ }^{\circledR}$ Architecture Council 
become an instrument of value. As a result of this work, the intent is to create a first version of the SG IMM for greater socialization in the community. Subsequent work products, as described in the previous section, are expected to be derived from SG IMM Version 1. Like all its work products, the GWAC realizes that the concepts, methods, and tools are subject to review and refinement. Section 4, "Roadmap for SG IMM v1.0," explains the path forward in greater detail.

\subsection{Collaboration Terminology}

This SG IMM applies to the information exchange interface between computer-based technology using electronic communication media to coordinate operation and achieve goals related to the electric power system. The computer-based technology may exist in devices, software applications, or systems that control or coordinate devices, applications, or other systems. Throughout the document, the term "automation component" is used to indicate an intelligent piece of the electricity system. This piece can be an intelligent device (e.g., breaker, water heater, distributed generator, transformer), a system (e.g., transmission control center, building energy management system, substation automation system) or a software application (e.g., supervisory control and data acquisition (SCADA), billing system, feeder load balancing program). The common denominators between all automation components are 1) they can execute software programs and 2) they can communicate with other automation components in the application of their programs.

Suppose two parties, Party A and Party B, decide to collaborate on an activity. To do this, they need to agree on the interaction process to support their activity, the information required at each step of the process, and the mechanism they will use to make this information flow between them. We refer to the concepts involved in this electronic interaction as a collaboration model.

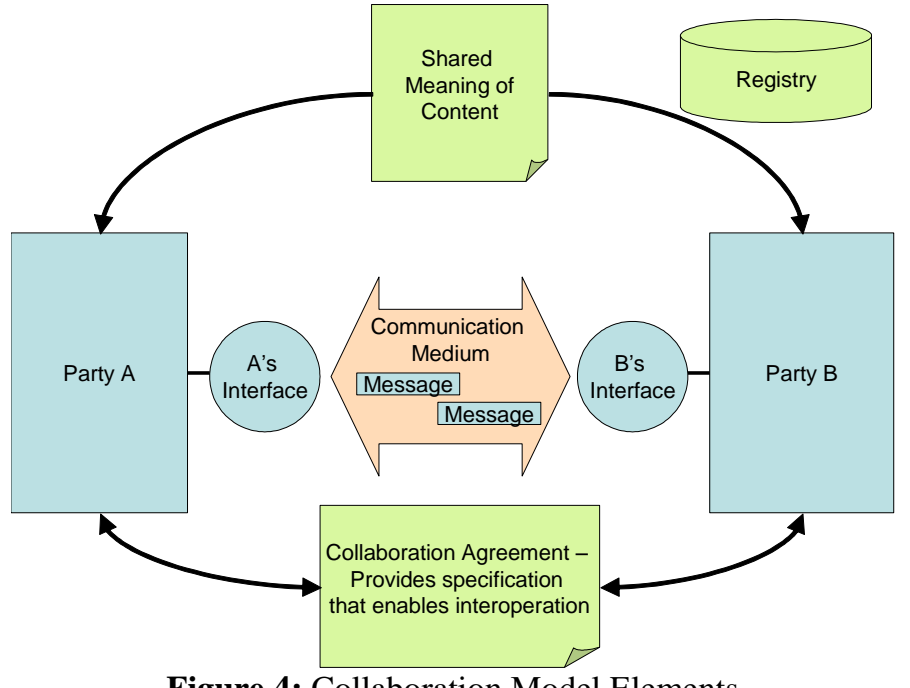

Figure 4: Collaboration Model Elements
As shown in Figure 4 (adapted from [9] and consistent with the Interoperability ContextSetting Framework), for any interaction to succeed, the parties involved must agree on several elements of communication. The elements of a collaboration model are described in a collaboration agreement; this agreement specifies the interface that each party exposes to the outside world. The interfaces send or receive messages containing information in a certain format (syntax) and with mutually understandable content. The data exchanged can be specified in an agreed-upon structured vocabulary that is common or shared between the two parties.

The collaboration agreement describes the roles and capabilities of the parties to achieve a shared outcome. It specifies the interface, the message definition, the message content supported between two transacting parties, and the expected response. The collaboration agreement explains what actions (services) its interface can perform, what format it expects in the message being communicated, what approach to make sure that the interaction is used, and the meaning of items contained in the message.

An interface is the point of contact that an automation component has with its interacting partners, and in particular, between communicating automation components. The interface describes the services that a

Page 15 of 46

The Smart Grid Interoperability Maturity Model is a work of the GridWise ${ }^{\circledR}$ Architecture Council 
party agrees to support, including error handling. Interfaces also specify the proper sequencing of information needed to affect an outcome. For example, before a switch can be opened, it must be selected for operation in a previous message exchange.

The message is the packet of information that is communicated between parties. Protocols specify the format of the message packet and can have several layers of communication-related information in the message header. For our purposes, we focus on the action or service requested and the message content (payload) related to the business at hand.

A shared vocabulary unambiguously defines the real-world concepts that are referenced in an information exchange. It provides a common language (shared meaning) about these concepts and their relationship to one another. Interacting parties commit to the shared meaning so that they can communicate about message content without necessarily committing to a globally shared theory of operation. These concepts may be called by different names because of various information-exchange implementations involving different approaches and protocols, but the shared meaning of content serves as a common point for interpretation.

A registry is a separate set of software that stores information about the automation components involved in an information exchange as well as aspects of the collaboration agreement itself. A registry is a separate repository that is shared by a community of interested parties. It is much like a telephone book, though the community can decide to strictly control access to the registry. Registries need not be centrally managed repositories, but can be distributed and divided into topics serving different needs. Parties can register their devices and interfaces with the registry. One can query the registry's repository to find information about registered subjects such as transacting parties and the communication mechanisms they support.

To apply the SG IMM, the interface of interest must be identified and all artifacts pertaining to the specification of the collaboration agreement should be available for review. 


\section{Structure of the SG IMM}

The following tenets have driven the form and structure of the SG IMM. The SG IMM should be able to encourage the maturity of interorganizational interoperability. It must be simple and straightforward to be usable and useful. While interoperability is complex, the maturity model should be organized to be readily understood at a high level, with details and complex issues being addressed as users move further into the model. The model should focus on the interfaces between interacting organizations' automation systems, understanding that while true interoperability extends into the implementations on either side of the interface, the interface collaboration agreement needs to reflect the expectations of all parties and consequences of failure to perform.

While interoperability is complex it is also difficult to define for some people. This is because interoperability is not an entity in and of itself - which is what can make it easily understood at a conceptual level yet difficult to describe. The problem is that shared conceptual understanding can differ in many ways between different people at a detailed level so a conceptual definition for interoperability is not good enough if a method is to be developed to measure it. Interoperability is a shared property of diverse entities that allows them to work together and it is the ability to work together that this model is designed to measure. This includes all aspects, from policy setting through design, documentation, operation, maintenance, security, quality of service and many other areas.

The structure of the SG IMM is based on the GWAC Interoperability Context-Setting Framework and it uses this framework to capture context in terms of relating the SG IMM to specific, measurable business and technical goals and then using these goals to provide further context in terms of metrics that can be used to assess interoperability maturity levels as it relates to these metrics. By collecting interoperability maturity assessments for multiple metrics, a picture of overall interoperability maturity can be built for a specific Interoperability Area.

\subsection{A Landscape for Exploring Goal Statements}

The GridWise Interoperability Context Framework is shown in Figure 5 and described in [5].

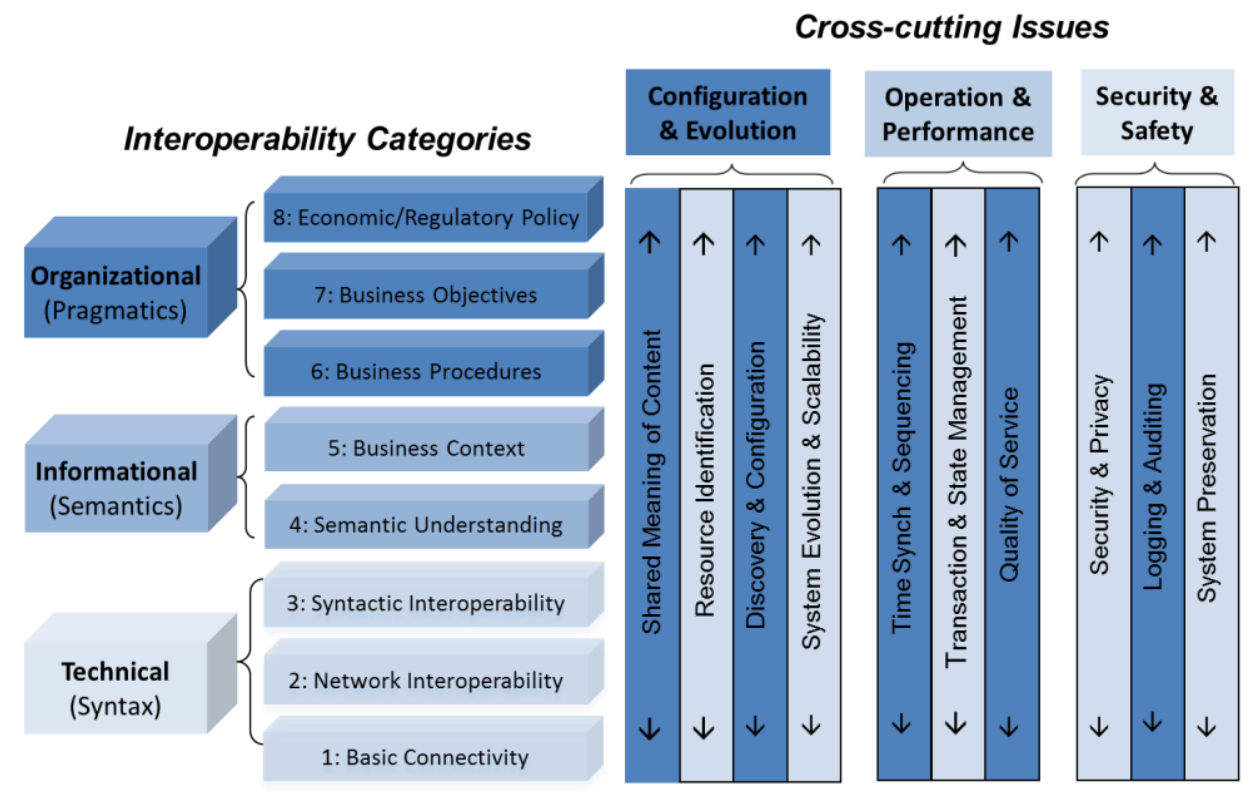

Figure 5: Interoperability Context-Setting Framework

Page 17 of 46

The Smart Grid Interoperability Maturity Model is a work of the GridWise ${ }^{\circledR}$ Architecture Council 
This framework builds upon the organizational, informational, and technical interoperability categories of the NEHTA model and identifies higher levels of granularity as inspired by other architectures. In addition, the framework identifies ten issue areas that cut across the interoperability categories. This provides a two-dimensional landscape that helps people as they focus on specific areas of concern.

To simplify and provide a high-level view of this landscape, the cross-cutting issues are proposed to be collected into three issue areas:

\section{- Configuration \& Evolution}

- Shared Meaning of Content

- Resource Identification

- Discovery \& Configuration

- System Evolution \& Scalability

- Operation \& Performance

- Time Synchronization \& Sequencing

- Transaction \& State Management

- Quality of Service

\section{- Security \& Safety}

- Security \& Privacy

- Logging \& Auditing

- System Preservation

Figure 6 presents a simplified interoperability landscape as a place of entry at which SG IMM can be applied. The detailed levels in both the Categories axis and the Issues axis are used to focus communities of users into specific Interoperability Areas. Similarly, as with the CMMI, goals are identified at high levels and at more detailed levels to encourage consistency while going deeper into specific Interoperability Areas, as discussed above.

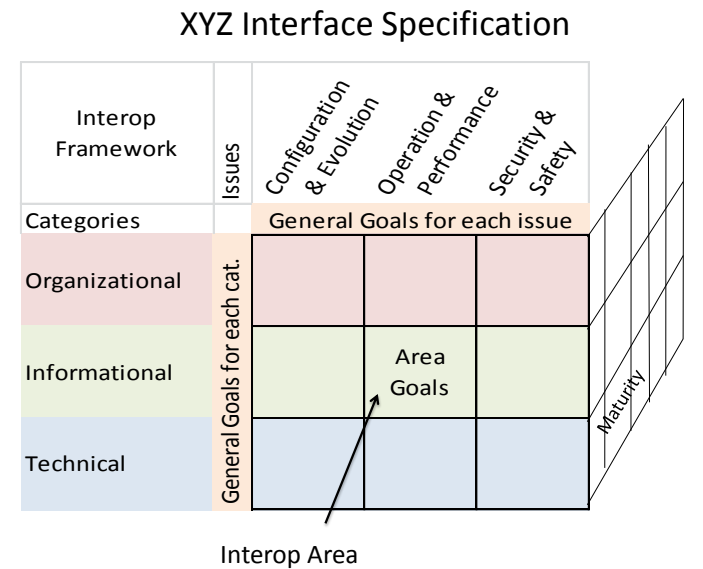

Figure 6: Simplified Interoperability Framework for SG IMM

The important thing to note here is that the SG IMM is designed to be applied for each Interoperability Area. In the above diagram, the Interoperability Area created by the intersection of the Informational level of the framework and the Operation \& Performance cross-cutting domain is one such area. 


\subsection{Interoperability Goals}

For successful advancement in building interfaces that engender interoperability, stakeholders must have specific goals. The SG IMM identifies high-level goals for each level of the framework and for each cross-cutting issue. Within the structure of the framework, these goals intersect in a matrix that allows the creation of more-detailed statements in support of the intersecting goals. The hierarchical nature of the model can thus also allow for general goals associated with the top-level areas and finer-grained goals for the lower tiers. Ideas for goals were contributed from the work in [5] and others. The goals used to derive the metric statements applied to assessing interface agreement maturity include the following:

\section{General Interoperability Category Goals}

- Organizational goals

- O1: Economic and regulatory interoperability policies are defined for the community.

- O2: Regulatory alignment exists across the community.

- O3: Policy provides incentives and removes impediments to enable interoperability.

- O4: Policy is current and maintained.

- O5: Business objectives of community participants are complementary and compatible.

- O6: Compatible business processes and procedures exist across interface boundaries.

- O7: Business interfaces are consistent with the business objectives.

- Informational goals

- I1: There is an information model relevant to the business context.

- I2: The information model that supports the business context is derived from one or more general information models relevant to the functional domain.

- Technical goals

- T1: Structure and format of information exchange are defined.

- T2: Information transported on a communication network is independent from the network protocols.

- T3: Management of a network between interacting parties is aligned.

- T4: Transport protocols used in specific exchanges are consistent.

- T5: A communications path exists for transparent and reliable exchange between interacting parties.

\section{General Cross-cutting Issue Goals}

- Configuration and evolution (CE) goals

- CE1: Information models (vocabularies, concepts, and definition) are agreed to by all parties.

- CE2: Where multiple-source information models exist, there are bridges between them.

- CE3: Semantics (information model) are captured independent of the technical interoperability categories.

- CE4: Resources can be unambiguously identified by all interacting parties.

- CE5: Resource identification management is defined.

- CE6: Discovery methods exist for interacting parties.

- CE7: Configuration methods exist to negotiate options or modes of operation.

- CE8: Parties can enter or leave without disrupting overall system operation and quality of service.

- CE9: Interface contracts between parties allow freedom of implementation.

- CE10: A migration path from older to newer versions exists.

- CE11: Capability to scale over time without disrupting overall system operation.

- $\quad$ Operation and performance (OP) goals

- OP1: Common understanding of quality of service, time, and scheduling exists.

- OP2: Time order dependency and sequencing are defined.

- OP3: Time synchronization requirements are defined.

$$
\text { Page } 19 \text { of } 46
$$


- OP4: Transactions and state management capability (atomicity, consistency, integrity, and durability) are defined.

- OP5: Performance and reliability expectations are defined.

- $\quad$ Security and safety (SS) goals

- SS1: Security policies (e.g., confidentiality, integrity, availability, and accountability) are defined, maintained, and aligned among parties.

- SS2: Privacy policies are defined, maintained, and aligned among parties.

- SS3: Risk is assessed and managed.

- SS4: Logging and auditing processes are defined among parties.

- SS5: Failures fail safe (health of system above individual components)

Progress on the ability to achieve goals will be more easily measured and encouraged as users delve into the areas of interoperability defined by the intersection of the interoperability framework levels with the cross-cutting issues, as discussed above. These intersections or "cells" take aspects from the general goals on each axis to compose appropriate metric statements for their area. For example, a high-level goal in the Informational category is that there is an information model relevant to the business context. Another high-level goal, this time for Operations and Performance cross-cutting issues, is that common understanding of quality of service (QoS), time, and scheduling exists. A more detailed metric can be stated where these goals intersect such that the business context information model includes elements that support time, scheduling, time synchronization, time order dependency, and sequencing requirements and mechanisms. Not every intersection of interoperability category goals with cross-cutting issues goals makes sense or has a reasonable chance of being measureable. The model strives to choose and compose metric statements with sensitivity to simplicity and usefulness, as described in the next section.

\subsection{Metrics to Measure Achievement of Goals}

One aspect to be aware of, but not explored in this document, is the potential for extending the SG IMM. There are many intersections, as described above, within any Interoperability Area, but not every intersection was utilized when generating these detailed goals. This was due to redundancy in goals because of similarity in some cases, but as application of the model increases and feedback is received there exists the potential to create additional detailed goals or even multiple detailed goals within each intersection. The decision to limit the current model to one or zero goals per intersection was to keep the size of the model manageable and avoid potentially confusing issues arising due to subtle differences in goals. Since the model is designed to be applied in the Interoperability Area, all of the detailed goals within an Interoperability Area form a pool of candidate goals to be applied in the SG IMM when it is used.

Once a detailed goal has been created, it is possible to generate a metric to test whether the goal has been met. For instance, for the above example:

- Detailed Goal: the business context information model includes elements that support time, scheduling, time synchronization, time order dependency, and sequencing requirements and mechanisms.

- Metric: the business context information model includes elements that support time, scheduling, time synchronization, time order dependency, and sequencing requirements and mechanisms.

In this example the metric simply restates the goal with the idea that evidence could be provided in a documented information model for examination to make an assessment of the appropriate level of maturity.

Page 20 of 46

The Smart Grid Interoperability Maturity Model is a work of the GridWise ${ }^{\circledR}$ Architecture Council 
Appendix A provides a list of 76 metric statements in a column organized by the 9 cells of the interoperability maturity model. These statements were distilled from nearly 200 goal statements that were developed by considering the cross-section of the general interoperability category goals and the general cross-cutting issue goals listed above. The original statements were reduced to remove redundant concepts, combine similar concerns, and consider aspects related to the goal that could be reasonably measured and for which evidence could exist.

\subsection{Levels of Interoperability Maturity}

Once the goals and associated metrics have been identified for the various parts of the SG IMM, the five maturity levels defined by CMMI can be applied to metrics to define levels of progress in terms of maturity. Interoperability areas of interest can be evaluated by focused teams (e.g., the maturity of the semantics about electricity usage captured in an information model applied to a building's electric meter interface). In addition, specific implementations may look broadly across multiple interoperability areas to better understand or characterize strengths and weaknesses with the objective of identifying priority activities for improvement.

Table 1: Criteria for Assessing Level of Maturity

\begin{tabular}{|c|c|c|c|c|}
\hline $\begin{array}{c}\text { Maturity } \\
\text { Level }\end{array}$ & $\begin{array}{l}\text { Community/ } \\
\text { Governance }\end{array}$ & Documentation & Integration & Test/ Certification \\
\hline $\begin{array}{r}\text { Level 1: } \\
\text { Initial } \\
\end{array}$ & $\begin{array}{l}\text { - management is } \\
\text { ad hoc }\end{array}$ & $\begin{array}{l}\text { - documentation is } \\
\text { ad hoc }\end{array}$ & $\begin{array}{l}\text { - integration is a } \\
\text { unique experience }\end{array}$ & - testing is ad hoc \\
\hline $\begin{array}{r}\text { Level 2: } \\
\text { Managed }\end{array}$ & $\begin{array}{l}\text { - managed by } \\
\text { project } \\
\text { agreement }\end{array}$ & $\begin{array}{l}\text { - documented in a } \\
\text { project } \\
\text { specification }\end{array}$ & $\begin{array}{l}\text { - integration is } \\
\text { repeatable, with } \\
\text { customization } \\
\text { expected }\end{array}$ & $\begin{array}{l}\text { - tested to plan with } \\
\text { results captured }\end{array}$ \\
\hline $\begin{array}{l}\text { Level 3: } \\
\text { Defined }\end{array}$ & $\begin{array}{l}\text { - managed by } \\
\text { community } \\
\text { agreement }\end{array}$ & $\begin{array}{l}\text { - references } \\
\text { community } \\
\text { standard with } \\
\text { some } \\
\text { customization }\end{array}$ & $\begin{array}{l}\text { - integration } \\
\text { repeatable with } \\
\text { predictable effort }\end{array}$ & $\begin{array}{l}\text { - tests exist for } \\
\text { community with } \\
\text { certification } \\
\text { - members claim } \\
\text { compliance with } \\
\text { standard }\end{array}$ \\
\hline $\begin{array}{r}\text { Level 4: } \\
\text { Quantitatively } \\
\text { Managed }\end{array}$ & $\begin{array}{l}\text { - processes ensure } \\
\text { currency and } \\
\text { interoperation }\end{array}$ & $\begin{array}{l}\text { - references a } \\
\text { community } \\
\text { standard w/o } \\
\text { customization }\end{array}$ & $\begin{array}{l}\text { - integration metrics } \\
\text { are defined and } \\
\text { measurements } \\
\text { collected } \\
\text { - reference } \\
\text { implementations } \\
\text { exist } \\
\end{array}$ & $\begin{array}{l}\text { - community test } \\
\text { processes } \\
\text { demonstrate } \\
\text { interoperability } \\
\text { - members claim } \\
\text { interoperable } \\
\text { conformance } \\
\end{array}$ \\
\hline $\begin{array}{r}\text { Level 5: } \\
\text { Optimizing }\end{array}$ & $\begin{array}{l}\text { - managed by a } \\
\text { community } \\
\text { quality } \\
\text { improvement } \\
\text { process }\end{array}$ & $\begin{array}{l}\text { - adopts an open, } \\
\text { community } \\
\text { standard }\end{array}$ & $\begin{array}{l}\text { - integration metrics } \\
\text { used for } \\
\text { improvement of the } \\
\text { standard }\end{array}$ & $\begin{array}{l}\text { - test processes are } \\
\text { regularly reviewed } \\
\text { and improved }\end{array}$ \\
\hline
\end{tabular}

To this end, the five maturity levels (described in Section 1.2) were used to define guidelines that can be used to assess interoperability maturity for specific metrics. The criteria are reflected in statements according to four characteristics of maturity: community/governance, documentation, integration, and test/certification. A pattern of progress is evident in the maturity statements for each characteristic as an evaluator moves through the maturity levels (see Table 1). The maturity statements are designed with the intent that they be supported by evidence in the interface collaboration agreement. However, not all maturity statements may apply to each metric statement. Future work on the SG IMM is expected to

Page 21 of 46 
more fully develop the mapping between metric and maturity statements to assist users with a consistent application of the model, including noting where some maturity statements may not apply to a specific metric.

An initial evaluation sheet can be constructed by considering each metric statement in the interoperability areas and measuring their interoperability maturity level using the maturity statements above. Such an evaluation sheet is depicted in Appendix A.

\subsection{Building the SG IMM}

Scoring methodologies were discussed early in the process of building the SG IMM with three different methods, identified as (1) Weighted Score Approach, (2) Pre-Qualification Approach, and (3) Baseline Approach. Regardless of which approach is ultimately pursued, the structure of the model, including the way that the metrics are organized with respect to Interoperability Areas, is identical. The differences in the scoring approaches appear in the methods and evaluation tools for applying the model. With this in mind, the focus for the initial SG IMM is on the development of goal, metric, and maturity statements in the structure described above.

The process for deriving this initial SG IMM follows a sequence described in the following steps:

1. Assess how best to leverage the context-setting framework.

2. Group cross-cutting issues into groups.

3. Develop high-level goals for each framework level.

4. Develop high-level goals for each cross-cutting issue.

5. Develop detailed goals for each intersection of (3) and (4).

6. Construct metric statements for the goals.

7. Create a matrix of maturity characteristics and maturity-level statements to provide guidance in assessing maturity for each metric.

8. Construct an evaluation sheet to apply the SG IMM and capture interoperability maturity for an interoperability area.

9. Assess scoring models.

The last step remains unfulfilled. Only crude checks of the evaluation sheet have been done for this beta version of the model. More thorough testing is needed with greater engagement by experts. Section 4, Roadmap for $S G I M M v 1.0$, describes plans for trials and greater participation in reviewing and refining the model.

While simple to outline, the process of developing goal and associated metric statements that can be assessed against the maturity statements is truly complex. The metrics need to cover the spectrum of concerns; however, redundancy and overlapping issues must be eradicated. In addition, terminology needs to be consistently applied in constructing the metric statements. While this initial model represents a reasonable attempt at constructing a model, only by applying the model and subjecting it to broad review can it be refined and improved. Figure 7 shows how these pieces come together conceptually in what is a very simple process to describe but that seemed to get longer with each step. 


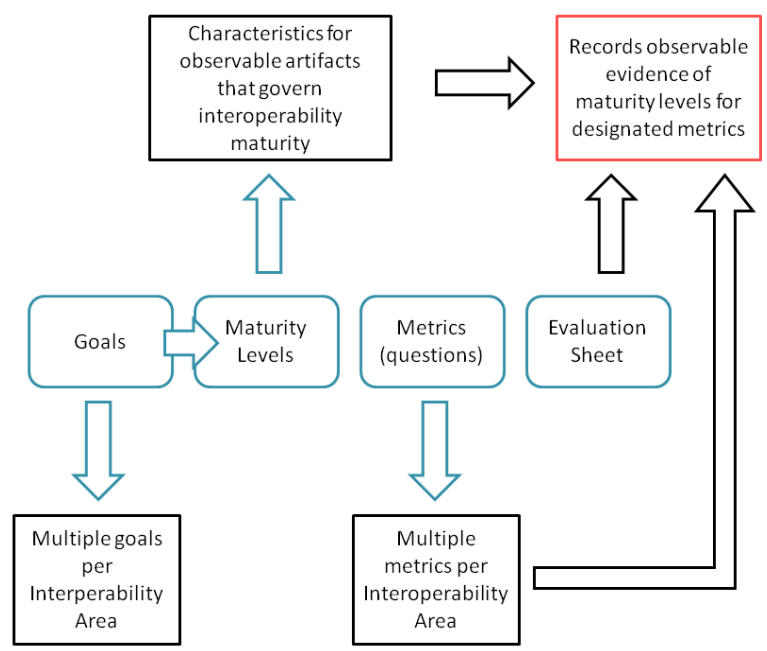

Figure 7: Process for Developing the SG IMM 


\section{Application of the SG IMM}

The NEHTA IMM provides a logical progression of activities for users of models like the SG IMM. These include the following steps:

- Define target: identify the interface between organizations to which the SG IMM is to be applied. For example, a dynamic pricing interface between a building and an electric service provider.

- Define domain: identify the stakeholders that form the boundaries of the targeted interface who need alignment behind a common set of objectives. For example, building operators, electric service providers, regulators, and product/system suppliers may be part of the domain for the example target above.

- Define goals: using the SG IMM, define the goals covering the interoperability areas that apply to the target interface.

- Identify practices: articulate the processes that are needed to support the interoperability area goals.

- Assess: gather evidence and use maturity model tools (such as scorecards) to evaluate the approaches for achieving interoperability.

Note, not all SG IMM metric statements are relevant for every smart-grid-related interface. Users may wish to focus on specific interoperability areas of an interface with the assistance of the SG IMM.

As has been done with CMMI, the desire of the SG IMM work is to develop a set of tools that can be applied to assessments, prioritization, and action plans. These could include scorecards, assessment and planning process diagrams, and education material (see Section 1.4, Audience for this Document).

\subsection{Sample Applications (Use Cases)}

Applications of the SG IMM will be as varied as the stakeholders in the current electric power system. The SG IMM can be considered for a range of applications. The common theme is that the application looks at specific interfaces between two or more information systems or devices.

Some example types of use cases are described below.

- Energy Market Operations. The typical use scenarios in energy markets focus on the transactions associated with buying and selling energy. This may involve the trading of energy generation resources in real-time, day-ahead or longer term timeframes. More recent offerings are bringing demand-response and ancillary service resources into the markets. Energy is also traded with bilateral agreements between various parties. In all cases, the interface between these players includes the exchange of information about price, schedule, quantity, and other attributes of the energy being traded. The markets also require clearing and settlement interfaces to provide a continuous and smooth market operation.

As a sample use case, a power exchange market uses the SG IMM as an assessment tool for understanding and developing an evolutionary roadmap for their trading platform. The assessment involves an internal team as well as an external, independent team under the sponsorship of the senior manager of the market system. The results identify a number of areas that could be improved. The senior manager and market members adopt an improvement program guided by the SG IMM recommendations. The outcome provides a more automated, efficient, and reliable mechanism for a new participant to join the market. The following scenario describes one of the resulting process improvements.

Page 24 of 46

The Smart Grid Interoperability Maturity Model is a work of the GridWise ${ }^{\circledR}$ Architecture Council 
An independent power producer (IPP) wishes to join the power exchange market and has completed the business and regulatory agreements to participate. The market has a standardized interface specification for automated participation in the market. The IPP builds or acquires the required technology to participate and completes a specified testing and certification program as defined by the market. The testing program exercises the actual interface to the market and exchange of test information in a market activity test scenario - i.e., the interaction does not actually touch any part of the operating market system. In the testing, the implementation has been evaluated for conformance to the market interface standard, including all other attributes identified in the SG IMM. Once certified, the IPP connects to the trading platform through a series of steps that include discovery of attributes by the IPP implementation and automated configuration of the IPP system to start trading.

- Retail Service Provider/Vendor Technology Community. Vendors of smart meters, retail service providers, retail customers, and integration partners form a specific community around a smart meter interface. One of the challenges of this community is to improve the standardization of the meter data interface and information exchange to the different metering application systems. A use case description for this community might be the following.

The community is loosely organized, with a number of different working groups focused on interoperability issues and standards. A stakeholder alliance, acting as a convening authority for the different groups, establishes a working group that uses the SG IMM to evaluate the impediments to achieving the interoperability goals of the industry. Using the SG IMM, the group discerns that the primary interoperability issues are at the organizational and regulatory level. Business goals of service providers, regulators, and vendors are not aligned for interoperability.

Using the recommendations developed during the SG IMM assessment, the utility members of the working group establish an action plan for refinement of existing standards to reduce ambiguity and minimize differing interpretations in the implementations. Further, they set a requirement that there be an independent test and certification authority. Finally, the group develops a set of guidelines for technology procurement processes to assist in evaluating the interoperability of specific vendor proposals. Metrics include conformance to the certification requirements of test and certification authority, as well as a series of evaluation questions to assess whether proposals meet the group's defined interoperability goals. This results in a dramatic improvement in integration of smart meters with metering application systems.

- A Multi-Player, Smart Grid Research Project. The smart grid vision holds the potential for radical change in the traditional business and operational processes associated with the electric power system. Research projects are testing new business models and new technologies. These projects can be done within an institutional structure such as an electric power market and their participants or they can be done by bringing together a set of electric service providers and vendors to develop new technologies and models and test them on a project basis. While the SG IMM is intended to be applied to communities, its use with an experimental project can be useful in evolving the resultant technology and business models into a permanent institutional structure. One of the first trials of the SG IMM (see Section 4, "Roadmap for SG IMM v1.0") is with such a research project aimed at developing a significantly more sophisticated business and technical model for energy market operations. The use case can be described as follows.

A group of utilities, several vendors, a research lab, and a transmission authority organize a project to determine whether a scalable communication signal about the price of energy can be used to modify overall system behavior. The signal provides a locational current price and

Page 25 of 46 
forecast of the actual cost of delivering electricity to any point in the system. In response, each utility may modify its own usage profile by initiating various demand-side load management programs such as demand response, distributed generation scheduling, etc., and communicates a response back into the system. The goal of the project is to develop the signaling technology to test whether this new distributed, price-based, control system can provide an efficiently scalable system for managing complex power flows and transactions.

The project has a strong focus on interoperability and decides to apply the SG IMM to assess potential improvements in system design should the project succeed and a larger community decide to implement it. In doing an SG IMM assessment, the project finds a number of potential design improvements such as better discovery and configuration capabilities for scaling. It also identifies a number of regulatory and business issues that would need to be addressed for the most efficient scaling of the technology.

- An Enterprise Smart Grid Application: Major investments are being envisioned or are currently underway that will result in advancement of grid functionality and empowerment of customers while institutionalizing operational efficiencies, integration of renewables and reduction of the carbon footprint. Smart grid capabilities require the integration of applications and systems that have typically operated in separate parts of an enterprise in the past. Hence, interoperability between myriad applications both within a utility enterprise and with its partners becomes the foundation or a prerequisite to deploying the next generation of operationally efficient and customer-facing services. This is not unusual, as many applications within a utility enterprise may have been developed independently of one another without the use of standards or longrange design for integration with other systems.

The SG IMM tool can be used to assess the interoperability maturity between these applications and systems or the organization at large, thereby enabling a utility to understand their gaps and risks and develop a roadmap that addresses these challenges. The results of the SG IMM can be applied to communicate interoperability needs to the executive management and to appropriate resources and funds to mitigate the risk for the organization. A use case driven from the perspective of a single utility enterprise might look like the following.

A smart meter rollout may not include the firmware (or firmware remote update capabilities) that would support bidirectional metering for distributed solar generation because that application was not anticipated at the time of the rollout decision. The consequence is that the meter data management and billing systems require that a new meter with appropriate firmware be installed for each solar generation installation.

The service provider is interested in understanding the lessons to be learned from the interoperability challenges of their smart meter rollout. They conduct an SG IMM assessment of the specific interfaces between a bidirectional metering application, the smart meter, the advanced metering system, and the billing system in order to better understand the architecture and design issues that caused the integration challenges to occur. The assessment includes a review of the regulatory, business and technology decisions as well as a review of the procurement requirements and specifications. The conclusion is that not enough attention was paid to the flexibility of the smart meter and advanced metering system to allow accommodation of use cases for system evolution and updates. Configuration and evolution immaturity are the primary issues, but the preparation of the rate case for approval also lacked a critical element of interoperability and standardization that would have anticipated the issues and addressed them.

The result of the SG IMM review is a better understanding of interoperability issues at the senior Page 26 of 46 
management level and with the regulator. Several SG IMM recommendations are added to future technology projects plans.

These are a few examples of applying the SG IMM. While hypothetical, they are based on real situations with practical assessment outcomes.

\subsection{Illustrative Example}

Imagine that an alliance of cell phone and mobile device manufacturers, cellular communications companies, and transportation authorities is formed to enable smart mobile device charging systems in airports, bus terminals, and passenger trains. Multiple vendors propose to offer charging kiosks where mobile device owners can connect their smart devices to charge for a fee. A smart device charging collaboration agreement is developed by the alliance which specifies the technical informational and organizational requirements for a device to connect to the charging station, obtain energy, and have the device owner's charge card billed for the service. An initial rollout of this service verifies the business proposition and now the alliance wishes to improve interoperability to enhance the user experience and attract more business. They engage their members in an SG IMM evaluation to better understand gaps and issues so that they can prioritize their efforts moving forward.

Some highlights from a hypothetical SG IMM assessment are presented below. They indicate interoperability areas in the interface specification where the alliance is quite mature and other areas where investments in standardization and testing appear valuable. The alliance maintains a series of documents that describe the interface specification and holds meetings quarterly to advance work items, review status, and address new issues.

Interoperability Area: Operation \& Performance - Technical

OP4-T1: Transaction and state management syntax is specified.

Metric Maturity Assessment: The interface specification references a power line carrier protocol and supported versions to be used for communicating with the charging kiosk. The transaction and state management model is well-specified by the power line carrier protocol standard and maintained by that community of organizations. A reference model exists for how the transaction model works and users can claim compliance through a certification authority. The experience of using the protocol and ease of implementation is not monitored, and improving the protocol further is being done on an ad hoc basis by the protocol community. This metric is deemed to be at Level 4 - Quantitatively Managed. It adequately addresses the needs of the smart device charging alliance, and further improvement may be out of their control.

OP3-T3: Time synchronization management between interacting parties is specified across the network. Metric Maturity Assessment: The interface specification references the openly available time format standard to be used and supported by the power line carrier protocol. It also specifies the timestamp that will be used in the message exchange and that the accuracy is to be good within 200 milliseconds in order to support the business processes including billing. While the alliance sponsors the testing of integration of smart devices with vendor charging kiosks, the tests presently do not explicitly cover time synchronization issues, instead smart devices are assumed to be synchronized to the required accuracy by their communications service providers and the internal clocks provided by the device manufacturers. Checking each maturity statement, the metric is found to be substantially at Level 3 - Defined; however, time synchronization testing and certification is noted as an area for improvement. As part of their prioritization and planning effort, the alliance may wish to review problem reports to see if issues are reported that may relate to this shortcoming.

Page 27 of 46

The Smart Grid Interoperability Maturity Model is a work of the GridWise® Architecture Council 
Overall Interoperability Area Maturity Assessment: The Operation \& Performance - Technical Interoperability Area's other metrics are evaluated and it is found that these aspects are generally documented, based on openly available standards, with governance procedures, and testing emerging at the community level. Testing and certification can be improved to make this assessment stronger and position the alliance to go to the next level. The overall score is Level 3 - Defined.

Interoperability Area: Configuration \& Evolution - Informational

CE1-I1: A business context information model supports the message definitions of the interface. Metric Maturity Assessment: The interface specification maintains an information model in UML. The message definitions themselves are defined in either XML Schema (extended mark-up schema language) or OWL (web ontology language) and a configuration option is provided in the negotiation business process between the smart device and the kiosk that indicates which format is used. The alliance supports a test procedure for individual products to check that their messages conform to the business context information model; however, some products are extending their products to use an emerging standard for exchanging customer's home address (should the customer opt in) so that special, non-charging related services can be provided through the interface. The interface specification does not cover how to address extensions and connectivity failures with some kiosks are being reported. The metric is assessed at Level 3 - defined; however, the problems with extending the business context model and testing are noted as areas that need to be addressed for fulfilling expectations at this level.

CE1-I2: The business context information model for the interface is derived from one or more general information models relevant to the functional domain. Metric Maturity Assessment: The business context model was built from specifications originated by the alliance. Some of the extensions being used by alliance members come from an international standard customer information model maintained by an international e-trade organization. The maturity level is 2 - Managed. An improvement to migrate the information model that is more widely recognized and maintained may be worthwhile to review as the alliance plans their next steps.

Overall Interoperability Area Maturity Assessment: In reviewing the other metrics for this Interoperability Area, the assessment is that the alliance is at Level 3- Defined. Aspects of information modeling can be improved by coordinating with other e-business groups that have similar customer information interactions; however, the model is well-defined, tested, and maintained to a level where members can claim compliance to the standards in this area.

Interoperability Area: Configuration \& Evolution - Organizational

CE4-O6, CE5-O6: Business processes are specified that support the mechanism for resource identification (creating and maintaining) to ensure unambiguous reference.

Metric Maturity Assessment: The alliance specifies that the unique identifier of the cell phone shall serve as the mechanism for identifying the resource being charged and linking that with the owner through the cell phone service provider. The cell phone identification scheme (electronic serial number of the phone, mobile identification number assigned to the phone, and system identification code for each carrier) is already in place and managed by the cellular communications community. The alliance has worked with the charging kiosk providers to develop a unique identification scheme for each kiosk. The alliance has decided that the cell phone owner will be billed for their charging by their carrier. When a cell phone connects to the charging kiosk, both sides exchange unique identifiers and these are used by the organizations back-office systems to support the billing and agreed to financial exchanges. This allows the kiosk to inform the customer of the rate for electric charging and for the communications company to bill the customer accordingly. While the business processes are specified, the kiosk identification scheme is unique to the alliance and restricted to the United States. The cell phone identification scheme appears

Page 28 of 46

The Smart Grid Interoperability Maturity Model is a work of the GridWise ${ }^{\circledR}$ Architecture Council 
to approach a maturity level of 5 - Optimizing; however, the kiosk identification is at level 3 - Defined. In addition, the present scheme restricts device charging to devices with built in cell phone features. The alliance wishes to review this approach so that non-cell phone, mobile devices can also use the kiosks.

CE10-O1, O3, O4, O5: Governance policy for a migration path from older to newer versions is specified. Metric Maturity Assessment: The interface specification for kiosk mobile device charging contains version identification. This is identified in the information model; however, the message exchange business process for checking the identifier is not specified. The result is that each company has developed their own approach to using the identifier and responding if the connection between mobile device and kiosk is supported. As a version of the interface is beginning to be rolled out, kiosk providers are working with mobile device providers on a case by case basis to establish connectivity and coexistence between newer and older versions of the interface specification. In addition, some new kiosk providers are only supporting the latest version of the specification. This is resulting in the inability of some mobile devices to use the charging service and dissatisfaction with customers. The assessment of maturity level statements ranges from level 1 - Initial, to level 2 - Managed. This is a clear area where the alliance may wish to prioritize their efforts.

Overall Interoperability Area Maturity Assessment: In reviewing the other metric statements in this Interoperability Area, the assessment finds that generally documentation and testing for compliance to interface specifications on configuration and evolution exist. Much if it is governed at the community level; however, a few significant shortcomings exist. Because of this, the overall maturity assessment is level 2 - Managed.

Interoperability Area: Safety \& Security - Organizational

SS2-O1, O4, T3: Privacy policy for the interface is specified, aligned, and managed among interacting parties. Metric Maturity Assessment: The alliance stipulates that each mobile device provider is to have established a privacy policy with their customers. A privacy policy template of concerns is maintained by the alliance and members are to verify that they meet a minimum set of requirements before being branded as certified for the mobile device charging program. While the privacy requirements are based on approaches put in place in other business relationships with consumers, there are emerging ecommerce standards that are not yet covered in the specifications for this area. The alliance is working on incorporating these privacy standards and meets regularly to review their progress. The maturity is assessed at level 3 - Defined as a policy is in place for the alliance and it is being managed by the community, but there is customization in its implementation.

SS5-O1, O4: Governance policy for identifying failsafe conditions and requirements is specified among interacting parties.

Metric Maturity Assessment: The alliance assumes that if a mobile device and a kiosk cannot establish a connection, then no charging will occur. Once a connection is established there is no documentation on what the interacting parties should do if communication is lost. As a result, some kiosks are periodically polling the mobile device and stopping charging immediately if there is no response, while others will continue providing the charging service until a minimum electric flow level is reached, indicating fully charged or disconnected. The consumer experience is different depending upon the kiosk vendor and reports of poor service or improper charges are occurring. The maturity level is assessed at level $1-$ Initial and the alliance more clearly understands that coordination is needed to improve interoperability in this area.

Overall Interoperability Area Maturity Assessment: The other metric areas in this Interoperability Area, especially in regard to well-documented security risk policies and procedures, are combined to indicate

Page 29 of 46

The Smart Grid Interoperability Maturity Model is a work of the GridWise ${ }^{\circledR}$ Architecture Council 
that the community is at level 2 - Managed, with several aspects at level 3 - Defined, and some shortcomings at Level 1 - Initial.

The result of the assessment indicates that the alliance is paying attention to interoperability and doing some things at a relatively high maturity level. It also highlights some areas where more work is needed. Some of these work areas appear to offer a significant improvement to interoperability maturity and require a minimum amount of coordination among the alliance members. Targeting effort in these areas could enhance the satisfaction of the customer in their mobile device charging experience. 


\section{Roadmap for SG IMM v1.0}

This initial document describing the concept and potential structure of an SG IMM provides a starting point for engaging the smart grid community on methods and tools for gauging and encouraging interoperability progress. It needs careful review and consideration, and a plan for revision based upon the comments and good ideas of reviewers across the base of stakeholders. Community engagement is important in order to gain widespread recognition, adoption, and use.

The following subsections describe a roadmap for developing an SG IMM Version 1.0 with an emphasis on stakeholder participation. The plan includes a period of trial applications of the SG IMM with real projects and communities of organizations interested in advancing interoperability. The plan also includes a workshop of experts in smart grid interoperability and process improvement mechanisms designed to discuss the objectives of the SG IMM, its use, and directions to move from a beta version to a Version 1.0. The results of the trials and workshop will then drive changes in the SG IMM to produce the first version of the model.

\subsection{Trials}

The SG IMM is in the process of early trials. Two such trials are described in this section. The process used to test the SG IMM in the trials includes the following steps:

1. Define target: identify the interface between organizations to which the SG IMM is to be applied. For example, a dynamic pricing interface between a building and an electric service provider.

2. Define domain: identify the stakeholders that form the boundaries of the targeted interface who need alignment behind a common set of objectives. For example, building operators, electric service providers, regulators, and product/system suppliers may be part of the domain for the example target above.

3. Define goals: using the SG IMM, define the goals and associated metrics covering the interoperability areas that apply to the target interface.

4. Identify practices and gather evidence: describe the processes and interface-related artifacts that support the interoperability area goals.

5. Assess: use SG IMM evaluation tools to evaluate the evidence and determine the interoperability maturity level. Note areas where the model is helpful and where there are problems or shortcomings.

6. Recommend improvements: review the assessment process to develop a set of recommendations to refine the model and propose ideas to enhance evaluation tools.

The locations of the trials presented below are coincidentally in the Pacific Northwest region of the United States. These trials represent different situations for advancing interoperability and should test different aspects of the model. Discussions are underway with others for additional participants from different regions in the early trial period. As communication about the SG IMM spreads, the GWAC hopes to solicit others in trials of the model.

\subsubsection{Pacific Northwest Smart Grid Demonstration Project}

The Pacific Northwest Smart Grid Demonstration Project (PNWSGDP) is the largest of the 16 Department of Energy funded Regional Smart Grid Demonstration Projects. The PNWSGDP is a unique demonstration across five Pacific Northwest states-Idaho, Montana, Oregon, Washington, and Wyoming. It involves more than 60,000 metered customers. Project participants represent a mix of public and private entities. The project started in November 2009 and runs for five years. 
Project Objectives: The Pacific Northwest Smart Grid Demonstration Project will

- validate new smart grid technologies and business models

- provide two-way communication between distributed generation, storage, and demand assets and the existing grid infrastructure

- quantify smart grid costs and benefits

- advance standards for "interoperability" (the smooth, seamless integration of all elements of the electric system) and cyber security approaches.

The key architectural concept that drives a consistent interface between systems in the project is a distributed decision-making approach based on current information delivered to each of the nodes in the system. This information consists of messages between the "upstream" and "downstream" nodes that communicate a value placed on a unit of energy (cents/kWh) at each node. The value of energy is forecast 72 hours ahead in increasingly coarse intervals. This is referred to as a transactive control interface.

Each node combines the information on energy value from the upstream and downstream nodes with its own information such as local weather forecasts, wind production, demand expectations, contract and regulatory requirements, etc., to determine the value and forecast for energy value at that node for the next 72 hours. It then determines whether it will make any operational changes, such as initiating a demand-response program, and communicates its own response signal upstream to the next node.

The nodes describe the interfaces between the participants and are the technical and informational levels of communication but also take into account the business and regulatory factors that determine local behavior of each utility in the system.

A simplified nodal structure for the project is shown in Figure 8. The transactive control interface specification (collaboration agreement) is the same for all nodes. All participants (the project community) need to implement this specification to interoperate in the system. A trial of the SG IMM is proposed to evaluate this interface specification. 


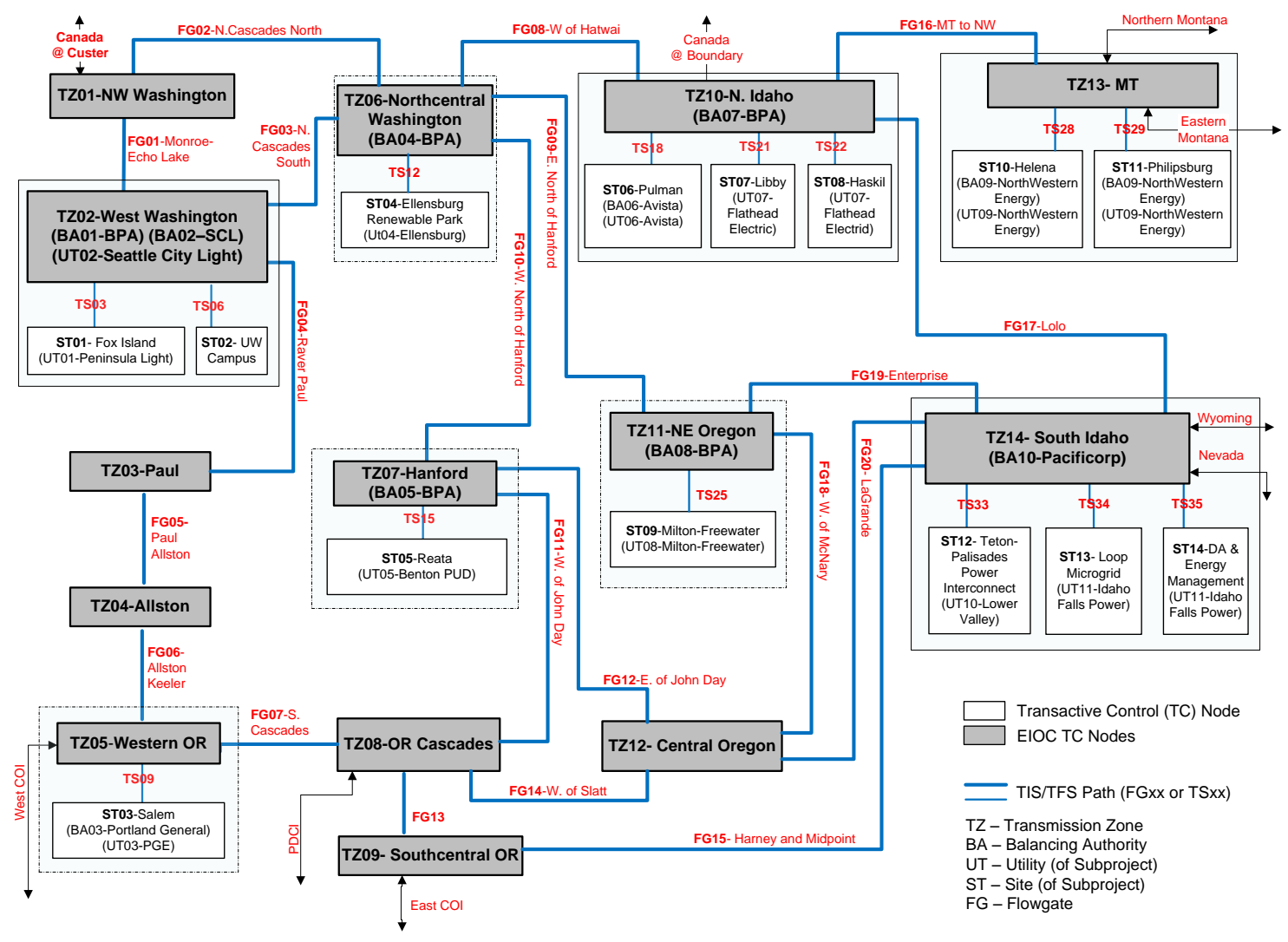

Figure 8: PNWSGDP Interacting Nodes and Logical Communication Structure

Each of the boxes represents one of the planned nodes in the system. The flowgates represent aggregated power flow channels across the boundaries between balancing authorities. The relative balance between the connected transmission zones and constraints through the flowgates can influence the value of energy at any time as represented by the transactive incentive signals associated with the respective transmission zones.

Another view of the system, Figure 9, shows the interfaces between the various system elements. The primary interface of interest is that between the Transmission Zone Nodes and the Utility/Site Nodes. This is referred to as the transactive control interface for the system.

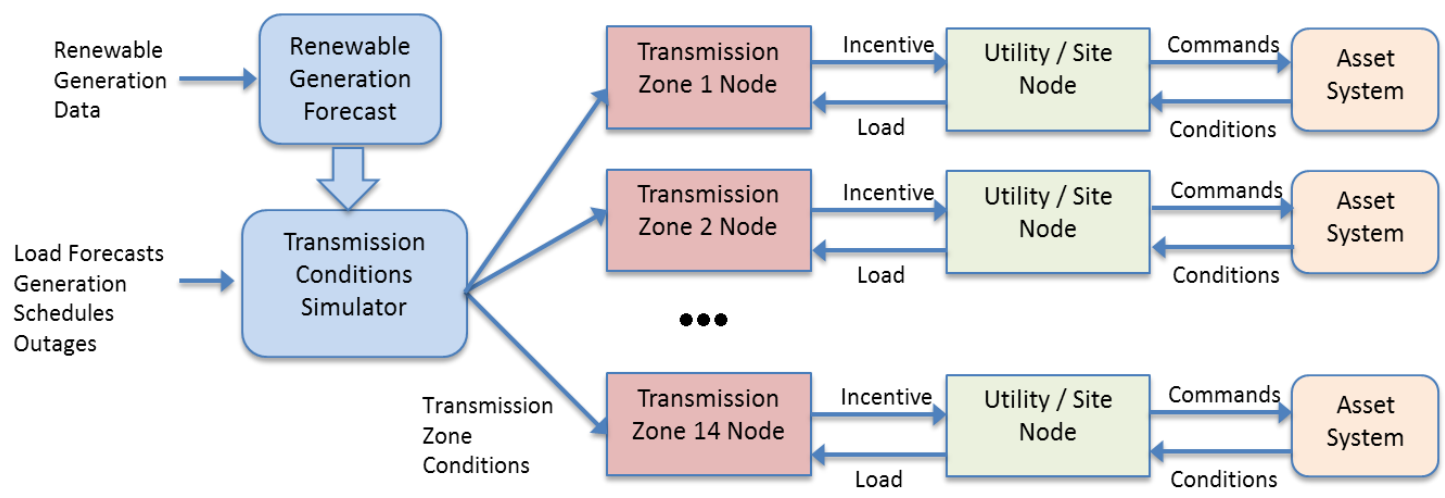

Figure 9: Transactive Signal Interfaces between Participants

Page 33 of 46

The Smart Grid Interoperability Maturity Model is a work of the GridWise ${ }^{\circledR}$ Architecture Council 


\section{PNWSGDP Trial Plans}

The objectives of the SG IMM trial for the PNWSGDP include the following:

- Test the overall process identified for an initial SG IMM evaluation.

- Evaluate the applicability of the metric statements in this case and select those statements relevant to the project.

- Develop a preliminary assessment of the project on the SG IMM and specify the process for refining the assessment.

- Provide insights and direction for further development of the SG IMM and the administration process.

The target interface is the transactive control interface that communicates energy cost and system response information between participants. The domain includes the interacting members of the project team representing their operating organizations.

\subsubsection{EWEB Project}

Eugene Water and Electric Board (EWEB) is a small customer-owned utility with 90,000 electric and 50,000 water customers. It is representative of a great many smaller to mid-size municipal utilities in the United States. EWEB is also striving to achieve a goal of meeting $100 \%$ of load growth through conservation. As a public utility, EWEB is committed to delivering more value to its citizens and desires to enable smart grid capabilities within its resource constraints and in the face of technical challenges such as its ability to integrate new technology with its the legacy systems. EWEB understands enough of these challenges to pilot the initial SG IMM evaluation tool because it brings them closer to addressing such challenges.

Project Objectives: Although the complexities and challenges facing smaller to mid-sized utilities may be smaller in scale, the implications of not addressing them are similar. This is where the SG IMM tool can play an active part. Using the beta version of the SG IMM tool, EWEB will hopefully gain insight into the current state of the interoperability maturity of the various interfaces that link a portfolio of application systems. The results of the SG IMM will be leveraged to identify the gaps in these interfaces, review them in light of their priorities, and develop an action plan to take a measured approach for the execution of its smart grid vision.

EWEB is launching a multi-year smart grid initiative that will necessitate procuring new automated metering (advanced metering infrastructure (AMI) and smart meters) and communication infrastructure and replacement of over 70 enterprise applications, some of which are home-grown while others are highly customized. The core applications, such as asset management, work management, mobile workforce management, customer information system, and financial system, provide the bulk of the functionality, while spreadsheets, proprietary code and batch jobs provide a wide array of scattered code that is equally important and drives the company's business. EWEB understands that the investment in new products and technologies requires a new approach to enterprise integration. There is a greater need for interoperability of the interfaces between the internal applications within its domain and of interfaces with applications of its partners, such as the Bonneville Power Administration.

A new AMI infrastructure will need to exchange information with legacy systems such as the customer information system (CIS), geographic information system (GIS) and the outage management system (OMS). It also needs to interact with a variety of homegrown and off-the-shelf but highly customized applications, such as two asset management systems and over two dozen spreadsheets that contain enterprise asset data. One or more of the interfaces with these systems will be used to try the SG IMM.

Page 34 of 46

The Smart Grid Interoperability Maturity Model is a work of the GridWise® Architecture Council 
In particular, a review of interfaces that depend on products and standards shared by others in the industry will be performed so that different levels of maturity can be explored.

\section{EWEB Trial Plans}

1. Define target: we identified four core interfaces within the organization appropriate for testing the SG IMM. These are between four applications, namely the new AMI meter head end, a new suite of asset management and work management applications and two legacy applications - the GIS and CIS. The specific interfaces were related to "Customer Interval Data," "Meter Change-out process" and "Asset Change."

2. Partner interfaces: there are no interfaces with other partners defined; however, the utility needs a capability to interface to its transmission service provider for power scheduling and to understand the generation capacity that will be leveraged in the market operation. These were considered in the roadmap.

3. Domain stakeholders: the key stakeholders are identified to be the executive management who drive the vision and strategy, product vendors who supply new products, internal subject matter experts who are intimately familiar with internal applications, and business and technical stakeholders.

4. Goals: the key goals are the interoperation of the new AMI with existing legacy applications for the named interfaces.

Hopefully, the trial of the SG IMM evaluation tool will provide a baseline and calibrated metrics through which EWEB can quantify the gap and mitigate their risk with appropriate investments for the desired outcome.

\subsection{Workshop Engagement and Outreach}

The idea for an SG IMM has been discussed within the GWAC for over a year and outreach to other interested parties in advancing interoperability indicates keen interest in developing methods and tools associated with such a model. These groups include the United States Department of Energy (DOE), including their smart grid program, and the many projects funded by the American Recovery and Reinvestment Act of 2009 (ARRA), as well as the SGIP that NIST established in November of 2009 to accelerate the development of smart grid standards. The Pacific Northwest Smart Grid Demonstration Project, described in the trials above, is one example of an ARRA SGIP project.

With this beta SG IMM, the GWAC can invite the participation of other interested parties in refining the concepts of the model, as well as developing usable methods and tools to meet the needs of the smart grid community. To this end, the GWAC expects to hold an SG IMM workshop of knowledgeable people from the diverse electricity stakeholder base. The purpose of the workshop would be to review the draft material, encourage comment, discuss improvements to the model, and engage the smart grid community in trials and adoption. The audience should include project representatives from DOE smart grid ARRA projects and experts associated with the SGIP effort. Besides needing to be a part of shaping the SG IMM, the participants can consider how derivatives of this model can be applied to a variety of concerns.

The desired result of a workshop would be the development of a Version 1 document for public comment and presentation at various venues. In addition, additional trials will be solicited and future meetings or workshops may be arranged to address actions resulting from the workshop. The ultimate aim is to continue to improve the SG IMM, and in the process develop a path for community adoption.

Page 35 of 46

The Smart Grid Interoperability Maturity Model is a work of the GridWise ${ }^{\circledR}$ Architecture Council 


\subsection{Adoption and Sustainment}

As the first version of the SG IMM develops, plans must be solidified with respect to the derivative work products targeted for use by stakeholders addressing interoperability concerns. Assessment scorecards, guides to use, checklists, and educational materials need to be considered and tailored for specific audiences.

The DOE smart grid ARRA projects represent an opportunity to obtain early feedback on the strengths and weaknesses of trying to apply SG IMM tools to specific efforts. These projects already have a stake in expressing how they are enabling interoperability. The DOE ARRA Smart Grid Demonstrations are asked to assess the "maturity level of interoperability realized between electricity system stakeholders.”[10]

Similarly, NIST and the SGIP are moving forward quickly on developing standards and guidelines to address priority smart grid concerns, such as dynamic pricing to end-users and electric vehicle integration [11]. The SGIP efforts may be able to make use of SG IMM methods and tools. In particular, the SGIP Domain Expert Working Groups (DEWGs) develop roadmaps that identify interoperability issues, actions underway to remedy the issues, gaps, and potential priorities. The SG IMM could become a helpful tool in a more systematic review of interfaces within a smart grid domain. In addition, efforts sanctioned by the SGIP for development of standards and guidelines may benefit from SG IMM tools as a means to focus efforts so progress can be made on specific items within the context of a greater perspective of concerns.

Other measurement methodologies and tools are needed to measure the progress of smart grid deployments themselves. The SGMM administered by the SEI is a management tool that electric utilities can use to plan, prioritize, and measure progress toward achieving the capabilities envisioned for a smart grid. The SG IMM aims to complement this work by focusing on the interfaces between smart grid devices and systems being deployed by service providers, energy suppliers, end-users and their product suppliers, and integrators. Representatives from DOE and the SEI have been involved in the SG IMM effort, and stronger coordination among the tools is being pursued with the realization of the complementary nature of these products. An organized suite of maturity model tools that assess and encourage the evolution of different aspects of smart grid deployments to higher levels of maturity may become a beneficial resource for the community.

\subsection{Relationship with Interoperability Testing \& Certification}

The SG IMM work products are meant to engender a culture of continuous improvement of interoperability within the smart grid community. As with quality assurance processes, people often equate this with testing and certification. While the creation of artifacts that help in the assessment of current practices is an important aspect of an improvement process and should contribute to assessing maturity, technical tests of specific implementations are only a contributor to the process of advancing interoperability. In fact, interoperability testing organizations operate most successfully when the system suppliers and integrators understand the entire lifecycle process in an interoperability enlightened way that respects clear articulation of technical, informational, and organizational agreements throughout the development process.

There is a two-way relationship between the SG IMM and the maturation of industry standards and associated certification test programs. With respect to standards themselves, the SG IMM may be useful in identifying an area for the development or improvement of standards. Use of the SG IMM can identify the most critical issues to address in order to achieve the level of interoperability that a standard should be expected to deliver to its community of users. Associated with advancing standards, the SG IMM also

Page 36 of 46

The Smart Grid Interoperability Maturity Model is a work of the GridWise ${ }^{\circledR}$ Architecture Council 
encourages the testing and certification of standards, particularly advancing testing from a project basis to one that involves a community of users.

A metric statement that is addressed by referencing a community supported standard that has testing authorities where product suppliers can claim compliance or certification will get higher maturity marks in an SG IMM assessment. To provide evidence of such product interoperability requires some combination of test tools and certification programs.

The SG IMM includes the maturity statements related to test and certification shown in Table 2.

Table 2: SG IMM Test and Certification Maturity Statements

\begin{tabular}{|c|c|c|}
\hline Maturity Level & Test/ Certification & \\
\hline Level 1: Initial & Testing is ad hoc & \\
\hline Level 2: Managed & $\begin{array}{l}\text { Tested to plan with results } \\
\text { captured }\end{array}$ & \\
\hline Level 3: Defined & $\begin{array}{l}\text { Tests exist for community } \\
\text { with certification }\end{array}$ & $\begin{array}{l}\text { Members claim compliance } \\
\text { with standard }\end{array}$ \\
\hline $\begin{array}{l}\text { Level 4: Quantitatively } \\
\text { Managed }\end{array}$ & $\begin{array}{l}\text { Community test processes } \\
\text { demonstrate interoperability }\end{array}$ & $\begin{array}{l}\text { Members claim } \\
\text { interoperable conformance }\end{array}$ \\
\hline Level 5: Optimizing & $\begin{array}{l}\text { Test processes are regularly } \\
\text { reviewed and improved }\end{array}$ & \\
\hline
\end{tabular}

The maturity of testing and certification processes is not presently covered in the SG IMM. Alliances that have testing and certification programs document and maintain their processes. They may review and gauge their programs according to standards for testing organizations (for example International Organization for Standardization (ISO) Standard 17025 for quality management of test laboratories) [12]. Reference to such a testing and certification regimen would be strong evidence for this aspect of maturity regarding a metric statement in an SG IMM assessment.

Advancement in the field of testing and certification processes is not a direct objective of the SG IMM; however, the work of groups, such as the Smart Grid Interoperability Panel's Testing and Certification Committee (SGTCC) can link nicely to this effort. The SGTCC Interoperability Process Reference Manual [13] is designed to assess a testing and certification program being used for a standard. Coordination of the work of the SGTCC in this area should be explored as the SG IMM work progresses to emphasize consistency of ideas and foster their complementary nature. 


\section{Conclusion}

An SG IMM can become the basis for assessing interorganizational interoperability processes and developing an improvement roadmap for maturing interoperability in the smart grid community.

A good deal of work that can serve as the basis for a model for smart grid interoperability maturity has already been done by respected groups. The approach leverages prior work by GWAC, the SEI, and NEHTA. Inspired by this material, an initial model for measuring interoperability maturity is proposed. Draft versions of the metric statements are organized according to interoperability categories and crosscutting issues as expressed in the GWAC Interoperability Context-Setting Framework. These are derived from goals statements inspired by the framework document. A series of maturity statements organized according to the maturity levels promulgated by the SEI's CMMI are presented as a tool to assess the status of each metric when applied to an interface specification.

Additional work needs to be done to establish the details of the SG IMM and improve the model. This will involve the participation of stakeholders in its review and refinement, and provide direction for its application. 


\section{Governance}

The SG IMM is a living, evolving set of material that influences the ongoing work of those involved in resolving interoperability issues related to the electric power system. The intent is to create derivative material to communicate effectively to multiple audiences whose participation is important to the advancement of interoperability in the electric system. A mechanism to correct, to update, and to clarify this model and its derivative material is necessary.

The GWAC shall maintain the SG IMM as reflected in Section 4, Roadmap for SG IMM v1.0, of this document; however, the material must consider the needs and views of the full range of stakeholders in an integrated view of the electric system. This requires the representation of various segments and a consensus-making process for decisions about update plans, actual revisions, and complementary material. A long-term governance plan for maintaining the SG IMM and related material needs to be established. Items to address in developing the governance for this material include the following:

- Establish a governance organization and populate it.

- Establish a revision control process.

- Establish a document posting policy.

- Consider web-based mechanisms to capture proposed changes, such as a controlled wiki.

- Establish metrics to measure successes and shortcomings of the SG IMM material and drive improvement. 


\section{Acknowledgements}

The creation of this document has been a collaborative effort of the GridWise Architecture Council. Particular recognition is given to the members of the Smart Grid Interoperability Maturity Model Team and supporting contributors: Stephan Amsbary, Rik Drummond, Tony Giroti, Doug Houseman, Mark Knight, Alex Levinson, Wayne Longcore, Randy Lowe, James Mater, Austin Montgomery, Terry Oliver, Phil Slack, Andreas Tolk, and Steve Widergren.

\section{References}

[1] GridWise Architecture Council, "Financial Benefits of Interoperability," prepared by Harbor Research, September 2009. Accessed November 2011 at http://www.gridwiseac.org/about/publications.aspx

[2] Widergren, S., A. Levinson, J. Mater, R. Drummond, "Smart Grid Interoperability Maturity Model," Proceedings 2010 IEEE Power and Energy Society General Meeting, July 2010.

[3] GridWise Architecture Council, "GridWise Interoperability Constitution Whitepaper, v1.1," December 2005. Accessed November 2011 at http://www.gridwiseac.org/about/publications.aspx

[4] GridWise Architecture Council, "Interoperability Path Forward Whitepaper, v1.0," November 2005. Accessed November 2011 at http://www.gridwiseac.org/about/publications.aspx

[5] GridWise Architecture Council, "GridWise Interoperability Context-setting Framework, v1.1," March 2008. Accessed November 2011 at http://www.gridwiseac.org/about/publications.aspx

[6] Software Engineering Institute, Carnegie Mellon University, "Smart Grid Maturity Model," Accessed November, 2011 at http://www.sei.cmu.edu/smartgrid/tools/

[7] Chrissis, M.B., M. Konrad, S. Shrum, CMMI: "Guidelines for Process Integration and Product Improvement," Second Edition, Addison-Wesley, 2007.

[8] National E-Health Transition Authority, Ltd, "Interoperability Maturity Model, v 1.0," March 2007. Accessed November 2011 at http://www.nehta.gov.au/component/docman/doc_download/220-interoperability-maturitymodel-v10

[9] IEEE P1547.3 “Guide for Monitoring, Information Exchange, and Control of Distributed Resources Interconnected with Electric Power Systems," IEEE Standards Association.

[10] United States Department of Energy National Energy Technology Laboratory, "Smart Grid Demonstrations - Recovery Act, Financial Assistance Funding Opportunity Announcement," CFDA Number 81.1222 Electricity Delivery and Energy Reliability Research, Development and Analysis, June 2009.

[11] National Institute of Standards and Technology, "Draft NIST Framework and Roadmap for Smart Grid Interoperability Standards, Release 2.0," NIST Special Publication for public comment, October 2011. Accessed November 2011 at http://www.nist.gov/smartgrid/

[12] ISO 17025. 2005. General Requirements for the Competence of Testing and Calibration Laboratories. ISO/IEC 17025:2005. International Organization for Standardization and International Electrotechnical Commission, Geneva, Switzerland. Accessed November 2011 at http://www.iso.org/iso/catalogue_detail.htm?csnumber=39883

[13] Smart Grid Interoperability Panel Testing \& Certification Committee, "Interoperability Process Reference Manual, Version 1.0,” November 18, 2010. Accessed November 2011 at http://collaborate.nist.gov/twikisggrid/pub/SmartGrid/SGTCCIPRM/SGTCC_IPRM_Version_1.0_Updated.pdf 


\section{Appendix A: SG IMM Evaluation Spreadsheet}

The following table represents an initial set of interoperability metric statements as rows organized by Interoperability Areas. The columns are a set of maturity statements organized by maturity level. Together they form a rough outline for performing an interoperability maturity level assessment focused on a specific interface. The letters that precede the metric statements provide traceability to the goal statements outlined in Section 2.2, "Interoperability Goals". The dark boxes indicate situations where a maturity statement may not be appropriate for a particular metric statement.

As a beta model, the statements are offered for review. Refinements are anticipated as a result of evaluation trials and future engagement with the smart grid interoperability community. Given the breath and complexity of concerns to achieve interoperability, future efforts may wish to go into a finer level of detail. These initial statements may expand into additional statements that investigate particular aspects that deserve attention. The GWAC expects discussion and feedback to drive refinement of the structure and detail of the model, thus expanding its usefulness. 


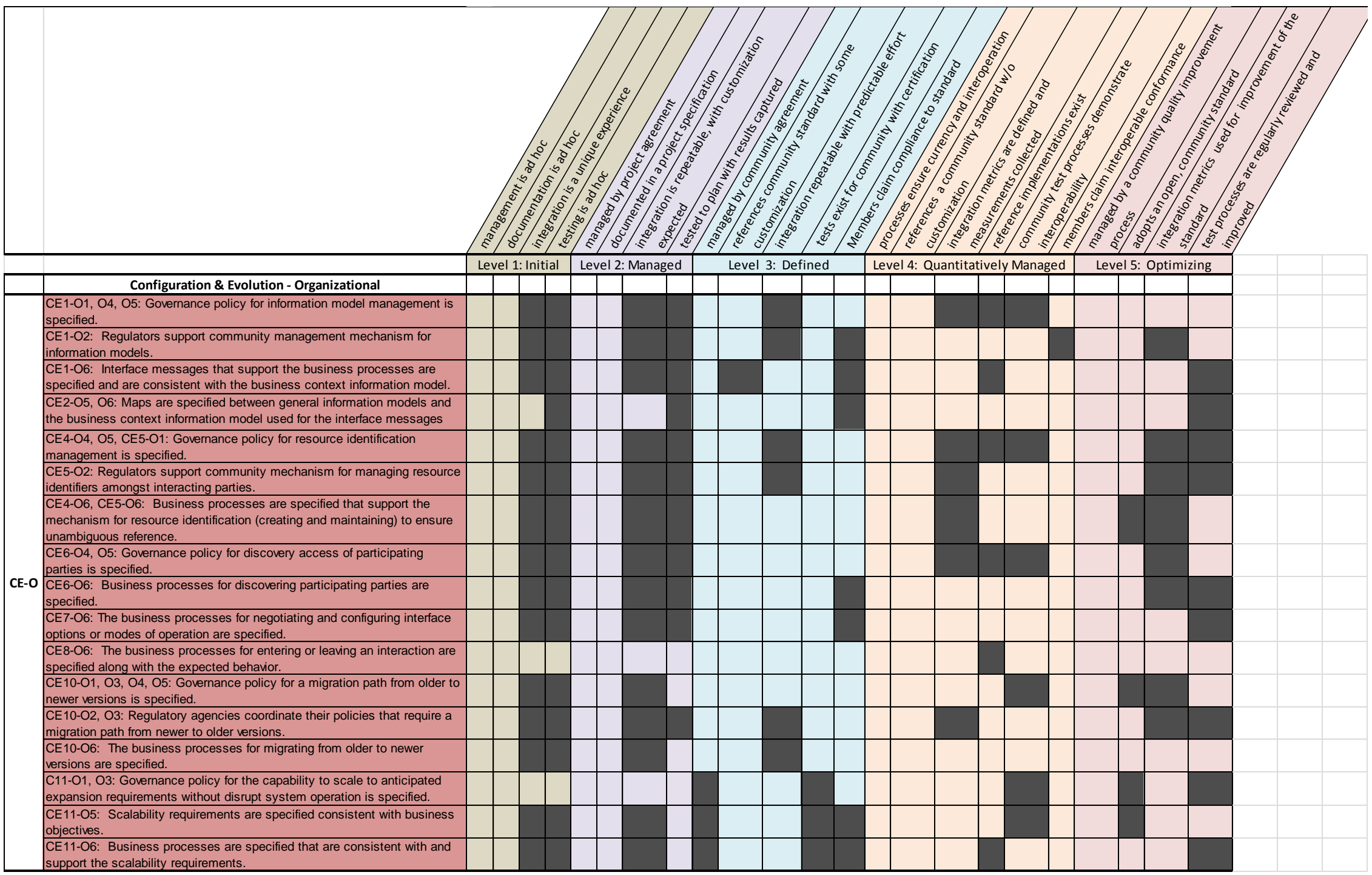

Page 42 of 46

The Smart Grid Interoperability Maturity Model is a work of the GridWise ${ }^{\circledR}$ Architecture Council 


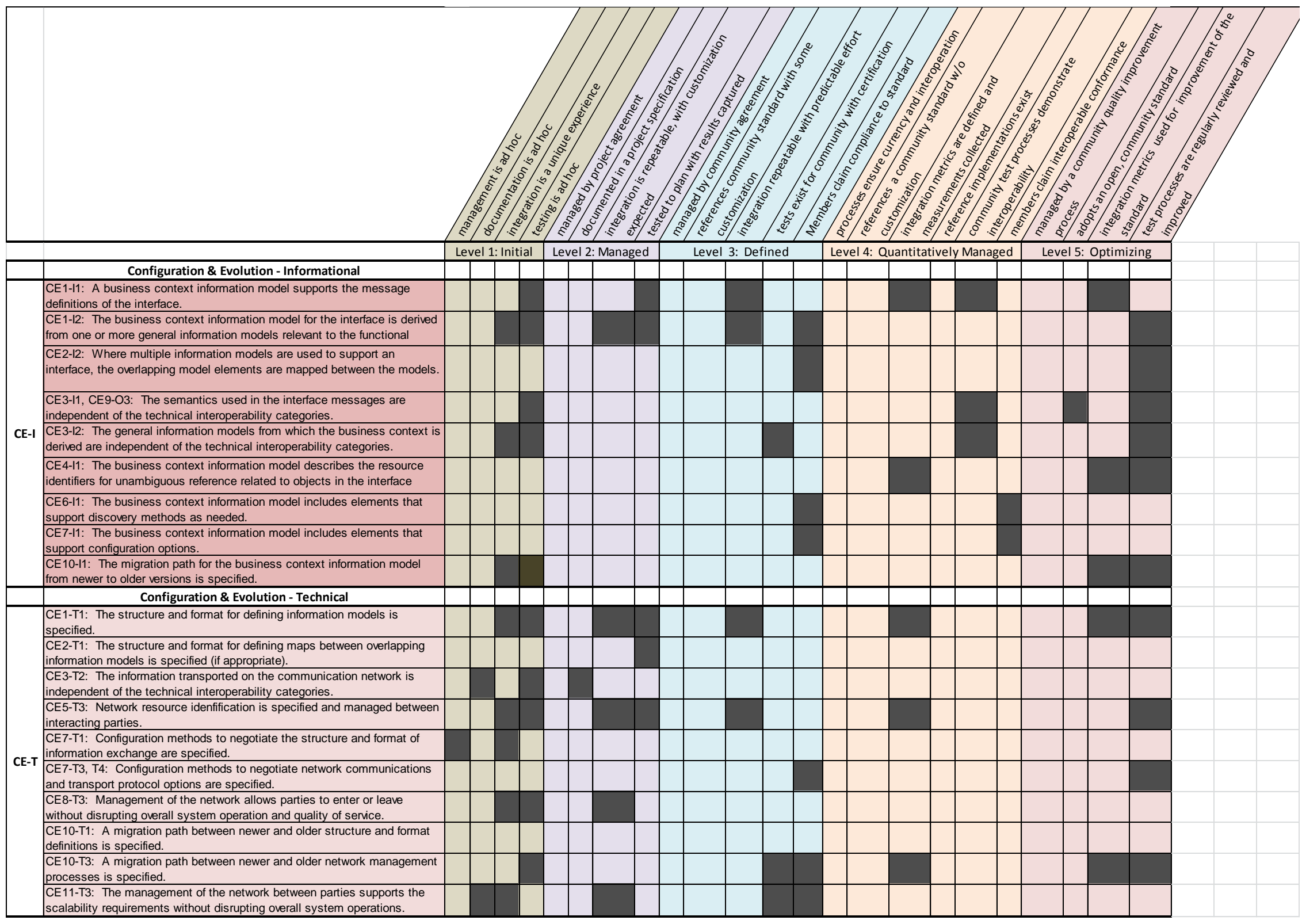

Page 43 of 46

The Smart Grid Interoperability Maturity Model is a work of the GridWise ${ }^{\circledR}$ Architecture Council 


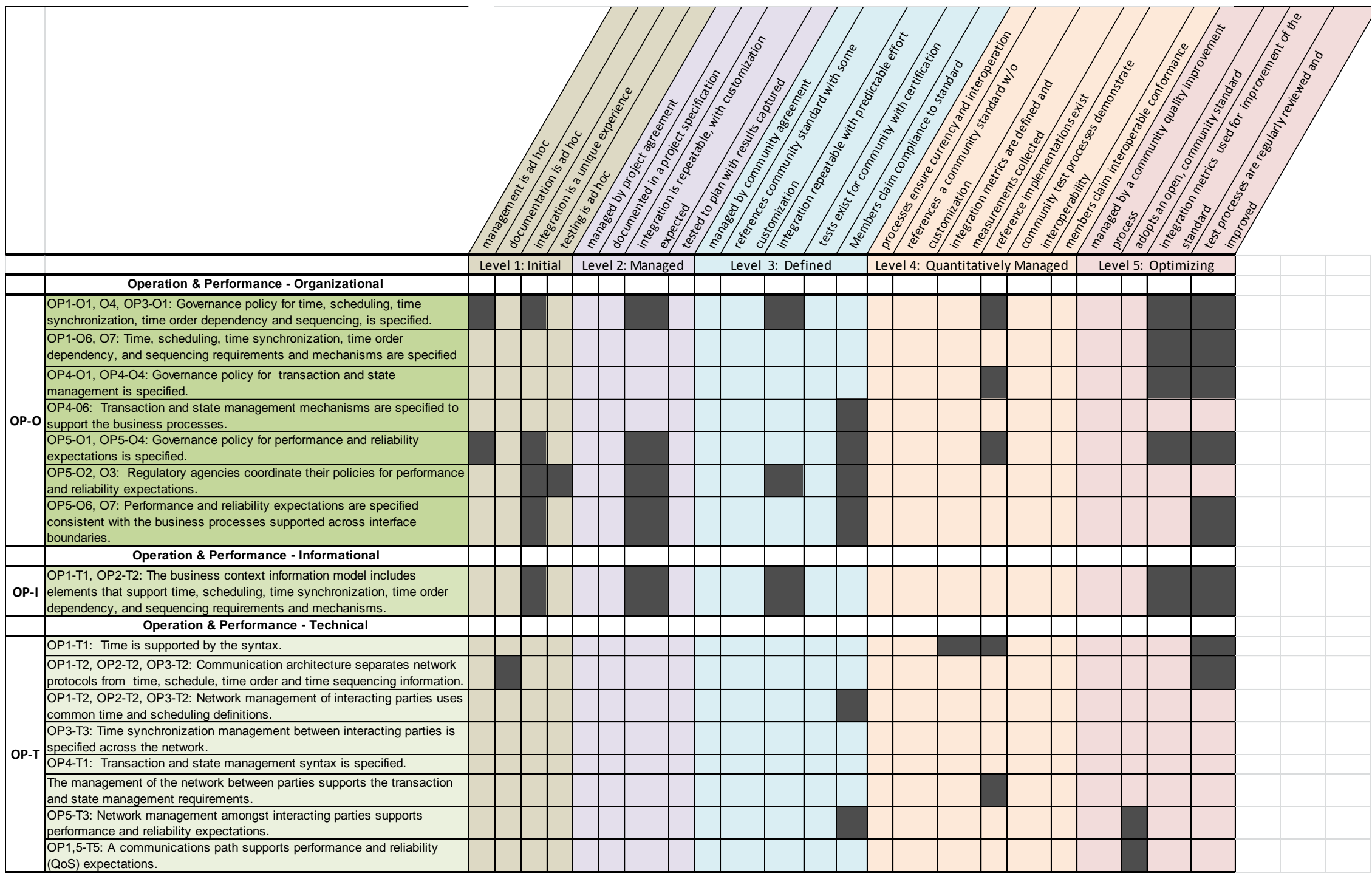

Page 44 of 46

The Smart Grid Interoperability Maturity Model is a work of the GridWise ${ }^{\circledR}$ Architecture Council 


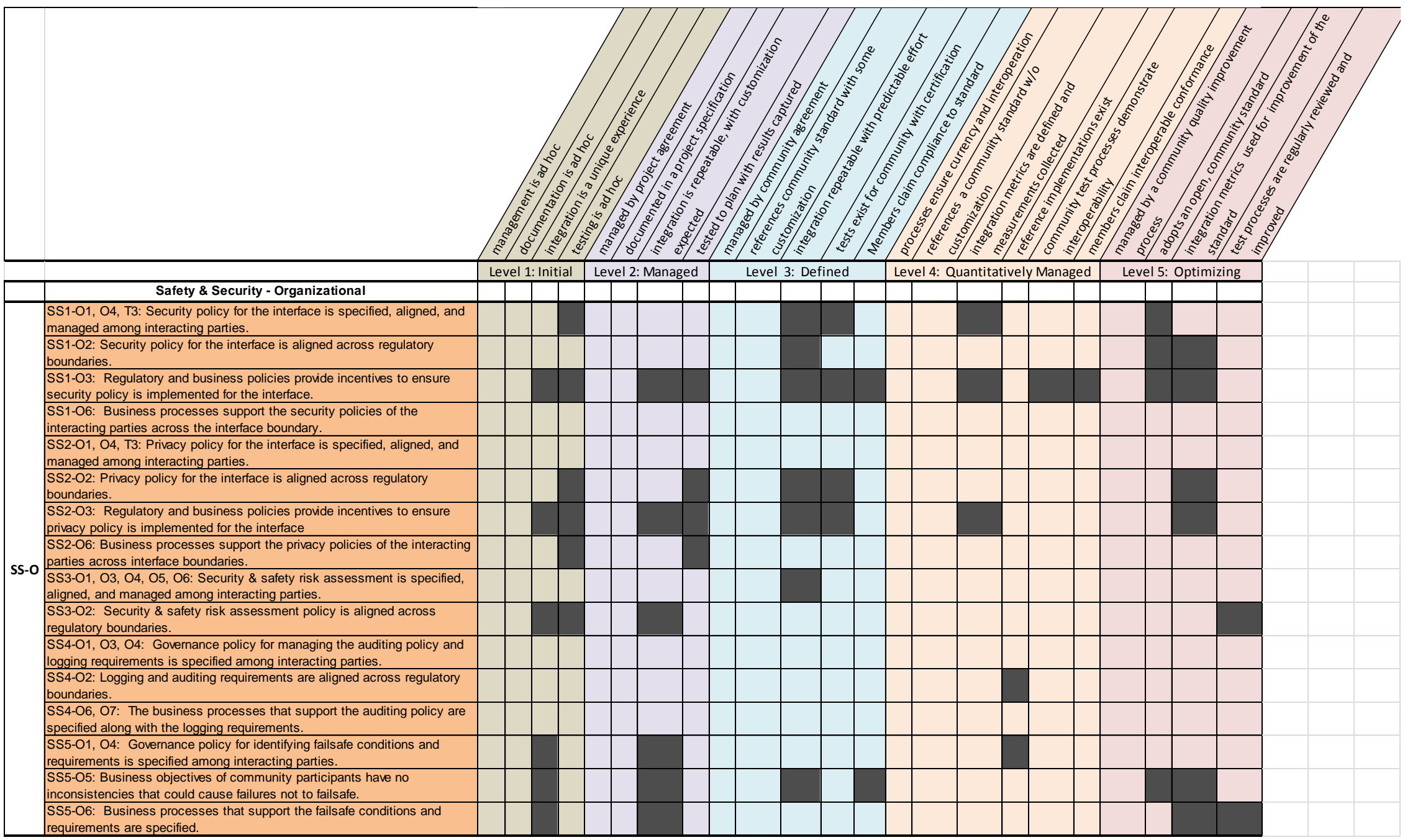

Page 45 of 46

The Smart Grid Interoperability Maturity Model is a work of the GridWise ${ }^{\circledR}$ Architecture Council 


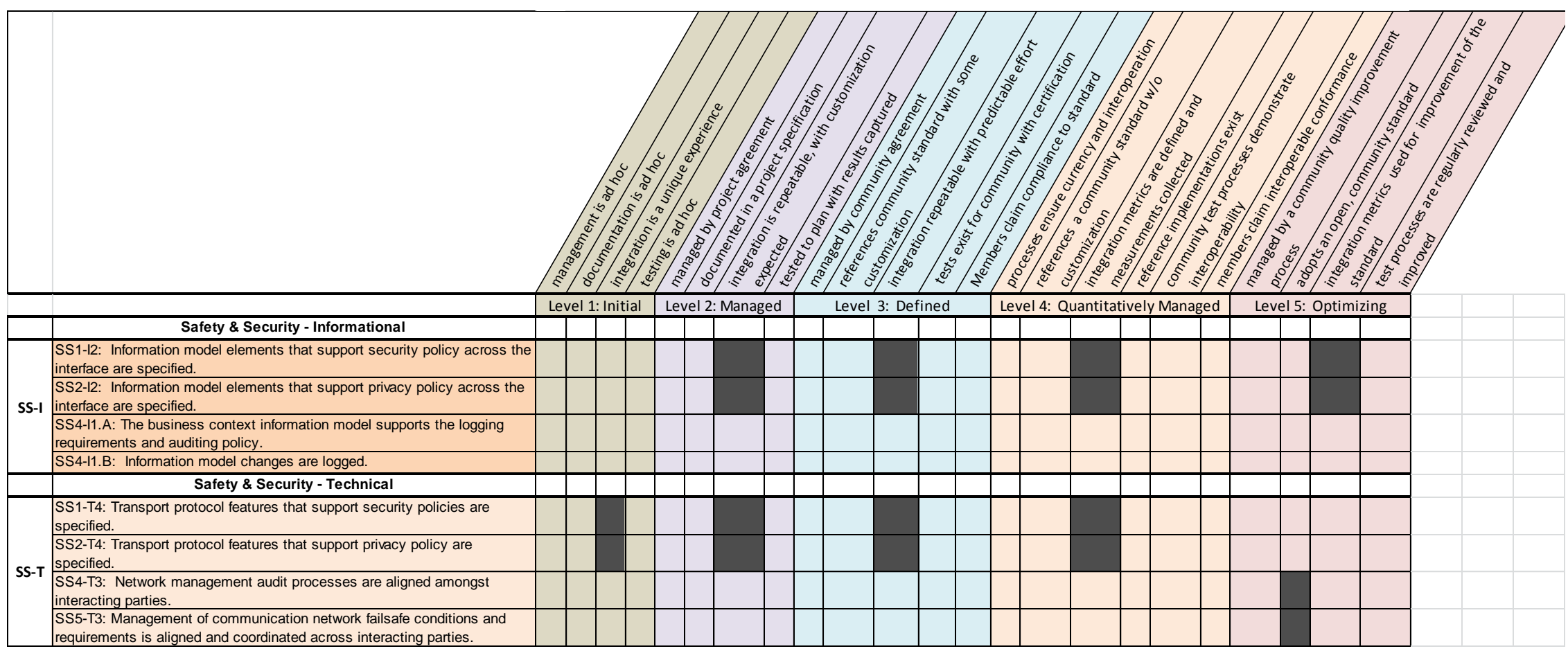

Page 46 of 46

The Smart Grid Interoperability Maturity Model is a work of the GridWise ${ }^{\circledR}$ Architecture Council 